\title{
HARMONIC ANALYSIS ON CENTRAL TOPOLOGICAL GROUPS
}

\author{
BY \\ SIEGFRIED GROSSER( ${ }^{(1)}$ AND MARTIN MOSKOWITZ $\left({ }^{2}\right)$
}

Introduction. In the present paper we continue and, in a sense, complete the investigations concerning [Z]-groups carried out in [8], [9], [10], and [11]; our notation and terminology is for the most part that employed in these papers. In particular, we utilize the following: $\mathscr{F}_{c_{\infty}}(X)$ and $\mathscr{F}_{b}(X)$ denote, resp., the continuous functions vanishing at $\infty$ and the bounded functions on the locally compact Hausdorff space $X, \varphi_{S}$ is the characteristic function of $S \subseteq X$. $G$ is a locally compact group; $M(G)$ and $\mathfrak{X}(G)$ denote, resp., the measure algebra and set of (this time) normalized characters of the elements $\rho$ of $\mathscr{R}_{\text {fin }}(G)$; here, $\mathscr{R}(G)$ denotes continuous irreducible unitary representations (or their equivalence classes) and $\mathscr{R}_{\mathrm{fin}}(G)$ those elements of $\mathscr{R}(G)$ which are finite-dimensional; in the case of a [Z]-group, they coincide. $M_{n}(C)$ is the matrix algebra of order $n$ over $C$. The symbol - denotes both topological closure and complex conjugation.

The results used here as well as the methods of proof depend primarily on those of [9], and only familiarity with [9] is actually presupposed. However, an overall perspective of the subject is provided in the introduction to [10]. As for harmonic analysis, another motivation for, and historical antecedent of, the present work is the program initiated by R. Godement in [3], [4], and [5], and carried out, in the more general case of [SIN]-groups, in [6]. The great detail and explicitness of the present work is of course due to the special position of $[Z]$-groups within the class of [SIN]-groups and, in fact, within all the classes of groups, satisfying compactness conditions, that have arisen to date. (For a full discussion of these classes see [10].) An excellent survey of the literature concerning harmonic analysis on groups, until rather recently, is provided in E. Hewitt [12], G. W. Mackey [17], [18], and I. E. Segal [24].

The paper is organized as follows. $\S 1$ contains approximation theorems (Theorem (1.2) and Corollary (1.4)) related to and dependent on those of [9]; however, in the

Received by the editors August 11, 1969.

AMS 1969 subject classifications. Primary 2220, 2265.

Key words and phrases. Central group, MAP-group, representations and representative functions, character formula, \#-operator, Fourier transform and inverse, uniqueness theorems, Plancherel Theorem, Riemann-Lebesgue Lemma, Bochner and Fourier Inversion Theorem, maximal ideal space of the group algebra and its center.

(1) Research partially supported by National Science Foundation grant GP-8496 and by Research Funds of the Graduate School of the University of Minnesota.

$\left.{ }^{2}\right)$ Research partially supported by National Science Foundation grant GP-5177.

Copyright (C) 1971, American Mathematical Society 
present case, the formulation is in terms of a fixed character of $Z(G)$ and approximation is uniform on $G$. This leads to a strengthened version (Theorem (1.5)) of the character formula of [9] eliminating the assumption of almost-periodicity. In $\S 2$ we extend the \#-operator defined in $\S 1$ of [9] from $\mathscr{F}_{c_{0}}(G)$ to the $L_{p}$-spaces and investigate its behavior with respect to convolution (Theorem (2.1)). Ideals in the group algebra $L_{1}(G)$ are \#-stable (Theorem (2.2)), and $L_{1}(G)$ is strongly semisimple (Theorem (2.4)) in the sense of Segal [22]. See also Hewitt [12].

In $\$ 3$ we define a new radical, larger than the strong radical-we call it the finite radical-and, using methods different from those of $\S 2$, we prove a result which both sharpens and generalizes Theorem (2.4) of this paper as well as Theorem (1.7) of [22]. This allows us to give a complete characterization of [MAP]-groups in terms of this stronger type of semisimplicity (Theorem (3.1)). The proof involves a uniqueness theorem for the Fourier transform of a measure (Theorem (3.2)). As a corollary another uniqueness theorem is derived for a modified notion of Fourier transform appropriate for central functions (Corollary (3.3)). The fact that the finite radical is in general larger than the strong radical is illustrated by Example (3.5).

$\$ 4$ is devoted to the derivation of the Plancherel Formula for [Z]-groups, with an explicit form for the Plancherel measure (Theorem (4.1)). Since this is one of the central results of the theory it may be appropriate to quote from the recent survey article by I. E. Segal [24] where the author states (p. 480): “The most important example, as well as the most significantly integration theoretic is that of the problem of the development of an effective common extension to the Plancherel Theorem for abelian groups and the Peter-Weyl Theorem for compact ones"; and again (p. 484), in reference to type I groups: “. . it is not to be expected that there exists any explicit expression for the Plancherel measure which is applicable to broad classes of groups." The point of $\S 4$ is to elucidate the fact that if the class involved is not too broad, i.e., $[Z]$, then both these problems have a common affirmative solution. A corollary, based on the results of $\S 3$, is a Plancherel Formula for central functions (Corollary (4.4)). Finally, as was mentioned in [9], compact groups can be characterized within $[Z]$ via the Plancherel Formula (Corollaries (4.5), (4.6)).

In $\S 5$ the equivalence criteria for representations derived in $\S 6$ of [9] are strengthened and generalized (Theorem (5.1)). There follow some facts (Corollary (5.2)) concerning the topology of the dual $\mathscr{R}(G)$ of the $[Z]$-group $G$. In Definition (5.3) and Theorem (5.5) a notion of "ampleness" of the group algebra of a locally compact group is introduced and studied. The presence of this property is shown to be equivalent to a certain property (Definition (5.8) and Theorem (5.9)) of sets of numbers on the unit circle, which in turn is equivalent to the conclusion of Theorem (5.1). An application to matrices (Corollary (5.10)) is given.

$\$ 6$ deals with the structure of the group algebra and its center in the case of a $[Z]$-group; the discussion utilizes much of the contents of the preceding sections. In Theorem (6.1) we show that if $G$ is an $[M A P]$-group then the center $\mathscr{Z}\left(L_{1}(G)\right)$ 
is a semisimple *-algebra consisting of central functions. In the special case of a [Z]-group the maximal ideal space $\mathscr{M}\left(\mathscr{Z}\left(L_{1}\right)\right)$ is found to be $\mathfrak{X}(G)$, the set of normalized characters of irreducible representations (Theorem (6.4)). Moreover, each ideal is $\vee$-stable, where $\vee$ denotes the usual involution (Corollary (6.5)). Theorem (6.6) describes the topological structure of $\mathfrak{X}(G)$, and Corollaries (6.7) and (6.8), respectively, generalize the Riemann-Lebesgue Lemma and the uniqueness theorem for the inverse Fourier transform of the abelian theory. In Theorems (6.10) and (6.11) we give generalizations of Bochner's Theorem and the Fourier Inversion Theorem, respectively. In the latter theorem the unique measure involved is naturally the Plancherel measure transferred to $\mathfrak{X}(G)$. This gives rise (Corollary (6.12)) to an isometry of the appropriate Hilbert spaces. Finally, we turn to the group algebra itself and its maximal ideal space $\mathscr{M}\left(L_{1}\right)$, the set of regular maximal 2 -sided ideals in $L_{1}$. It consists precisely of the kernels of Fourier transforms of the elements $\rho$ of $\mathscr{R}(G)$ (Theorem (6.18)). From this it follows that each ideal in $\mathscr{M}\left(L_{1}\right)$ is $\vee$-stable and of finite codimension (Corollary (6.20)). The above also yields (Corollary (6.19)) bijective maps, with a commutative diagram, between the following sets:

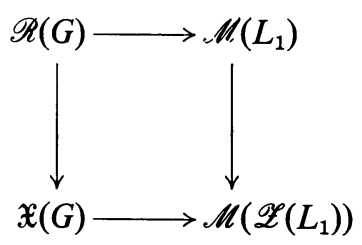

In the case of Hilbert space representations of $G$ and regular maximal ideals of $L_{1}$, the above results apparently generalize those of Kaplansky [14] in the case $G=A \times K$.

In fact, many of the results of the present paper are generalizations to [Z]-groups of questions raised and results stated for groups of the form $A \times K$ in [5].

1. Approximation theorems and a character formula. In order to prove the Plancherel Formula referred to above as well as for other purposes it is desirable to study the function space $\mathscr{F}(\chi)$ where $\chi$ is a fixed character in $Z^{\wedge}$ and $\mathscr{F}(\chi)$ denotes the linear space of complex-valued measurable functions on the $[Z]$-group $G$ satisfying the condition

$$
f(x z)=\chi(z) f(x)
$$

for all $z \in Z$ and almost all $x \in G$. In the case of a continuous function condition (i) holds for all $x \in G$ and all $z \in Z$. We will denote by $\mathscr{F}_{r}(\chi)$ the subspace of $\mathscr{F}_{r}(G)$ associated with representations in $\lambda^{-1}(\chi)$, where $\lambda: \mathscr{R}(G) \rightarrow Z^{\wedge}$ denotes the canonical map of $[9, \S 6]$.

Proposition (1.1). Let $G$ be a $[Z]$-group. Then $\mathscr{F}_{r}(G) \cap \mathscr{F}(\chi)=\mathscr{F}_{r}(\chi)$. 
Proof. If $f \in \mathscr{F}_{r}(\chi)$ then $f$ is of the form

$$
f=\sum_{k=1}^{n} \sum_{i, j=1}^{\operatorname{deg} \rho^{k}} c_{i j}^{k} \rho_{i j}^{k}
$$

where $\rho^{k} \in \lambda^{-1}(\chi)$, for $k=1, \ldots, n$. If $\rho \in \lambda^{-1}(\chi)$ then evidently $\rho(x z)=\chi(z) \rho(x)$, for all $x \in G$ and $z \in Z$, and hence $\rho_{i j} \in \mathscr{F}(\chi)$, for $i, j=1, \ldots, d_{\rho}$. Thus $f \in \mathscr{F}(\chi)$ $\cap \mathscr{F}_{r}(G)$. To prove the reverse inclusion suppose $f$ belongs to $\mathscr{F}(\chi)$ and satisfies (ii) where the $\rho^{k}$ are arbitrary elements of $\mathscr{R}$. Then $f=\sum_{k=1}^{n} f_{k}$, where $f_{k}=\sum_{i, j=1}^{\operatorname{deg} \rho_{i j}^{k}} c_{i j}^{k} \rho_{i j}^{k}$, and $\rho^{k} \in \lambda^{-1}\left(\chi_{k}\right)$, where $\chi_{k} \in Z^{\wedge}$, for $k=1, \ldots, n$. We may assume $f_{k} \neq 0$, for all $k=1, \ldots, n$. Since the $\rho^{k}$ are distinct, i.e., unequivalent, this implies (see, for example, Proposition (3.1) of [9]) that the $f_{k}$ are linearly independent; but, since $f_{k} \neq 0$ for all $k, f \neq 0$. Now

$$
f(x) \chi(z)=f(x z)=\sum_{k=1}^{n} f_{k}(x z)=\sum_{k=1}^{n} f_{k}(x) \chi_{k}(z) .
$$

The last term may be rewritten as $\sum_{s=1}^{n_{n}} g_{s}(x) \chi_{s}(z)$ where $\left\{\chi_{s} \mid s=1, \ldots, n_{*}\right\}$ is the subset of $\left\{\chi_{1}, \ldots, \chi_{n}\right\}$ consisting of distinct characters, and each $g_{s}$ is a sum of certain $f_{k}$ 's (or possibly an $f_{k}$ itself). Thus

$$
f(x) \chi(z)=\sum_{s=1}^{n_{s}} g_{s}(x) \chi_{s}(z), \text { for all } x \in G, z \in Z .
$$

Since the $\chi_{s}$ are all distinct and therefore linearly independent we conclude from the dependence relation (iii) that either $f=0$, or one of the $g_{s}=0$, or $\chi=\chi_{s}$, for some $s=1, \ldots, n_{*}$. Since the first alternative is false and the second implies, by linear independence, that one of the $f_{k}=0$, and thus is also false, we conclude that $\chi=\chi_{k}$, for some $k=1, \ldots, n$.

The proof now goes by induction on $n$. If $n=1$ then $f=f_{1} \in \mathscr{F}_{r}\left(\chi_{1}\right)$. Since $\chi=\chi_{1}$ we have $f \in \mathscr{F}_{r}(\chi)$. Now write $f=f_{1}+\sum_{k=2}^{n} f_{k}$ and assume $\chi=\chi_{1}$. Then $f(x z)-f_{1}(x z)$ $=f(x) \chi(z)-f_{1}(x) \chi_{1}(z)=\left(f-f_{1}\right)(x) \chi(z)$. Since $f-f_{1} \in \mathscr{F}(\chi) \cap \mathscr{F}_{r}(G)$, the inductive hypothesis tells us that $f-f_{1} \in \mathscr{F}_{r}(\chi)$. Since $f_{1}$ is in $\mathscr{F}_{r}(\chi)$ so is $f$.

The followirg approximation theorems amplify the assertions and utilize some of the methods employed in Theorems (3.2) and (3.3) of [9].

THEOREM (1.2). Let $G$ be a $[Z]$-group, $\chi \in Z^{\wedge}$ and $f \in \mathscr{F}_{c}(G) \cap \mathscr{F}(\chi)$. Then $f$ can be approximated uniformly on $G$ by functions in $\mathscr{F}_{r}(\chi)$.

Proof. Since $f$ is continuous and in $\mathscr{F}(\chi)$ we have condition (i) satisfied for all $z$ in $Z, x$ in G. Choose $\rho$ in $\lambda^{-1}(x)$ according to Theorem (5.5) of [9]. As remarked above, we have $\rho_{i j} \in \mathscr{F}(\chi) \cap \mathscr{F}_{c}(G)$, for all $i, j=1, \ldots, d_{\rho}$. Now $f(x z) \rho_{1 i}(x z)^{-}$ $=f(x) \chi(z) \rho_{1 i}(x)^{-} \chi(z)^{-}=f(x) \rho_{1 i}(x)^{-}$. Thus $f \rho_{1 i} \in \mathscr{F}_{c}(G / Z)$, for each $i$. Hence, by Theorem (3.2) of [9] (or by the Peter-Weyl Theorem), for any $\varepsilon>0$ there exists, for each $i$, a representative function $r_{i}$ associated with representations in $\mathscr{R}(G / Z)$ so 
that $\left\|f \rho_{\overline{1 i}}-r_{i}\right\|_{G / Z}<\varepsilon$; that is to say $\left\|f \rho_{\overline{1 i}}-r_{i}\right\|_{G}<\varepsilon$, for all $i$. This in turn yields the fact that $\left\|f \rho_{1 i} \rho_{1 i}-r_{i} \rho_{1 i}\right\|_{G}<\varepsilon\left\|\rho_{1 i}\right\|_{G}$. But then

$$
\begin{aligned}
\left\|f-\sum_{i=1}^{d_{\rho}} r_{i} \rho_{1 i}\right\|_{G} & =\left\|f\left(\sum_{i=1}^{d_{\rho}} \rho_{1 i} \rho_{1 i}^{-}\right)-\sum_{i=1}^{d_{\rho}} r_{i} \rho_{1 i}\right\|_{G} \\
& \leqq \sum_{i=1}^{d_{\rho}}\left\|f \rho_{1 i} \rho_{1 i}-r_{i} \rho_{1 i}\right\|_{G}<\varepsilon \sum_{i=1}^{d_{\rho}}\left\|\rho_{1 i}\right\|_{G} \leqq \varepsilon d_{\rho},
\end{aligned}
$$

because each $\left\|\rho_{1 i}\right\|_{G} \leqq 1$.

Since $\varepsilon>0$ is arbitrary and $\rho$ is fixed, the proof will be complete if we can show that $\sum_{i} r_{i} \rho_{1 i} \in \mathscr{F}_{r}(\chi)$. To this end it is sufficient to show that if $\sigma \in \lambda^{-1}(1)=\mathscr{R}(G / Z)$ and $\rho \in \lambda^{-1}(\chi)$, then $\sigma_{i j} \rho_{k l} \in \mathscr{F}_{r}(\chi)$, for all $i, j, k$, and $l$. For $x \in G$ and $z \in Z$, we have $\sigma_{i j} \rho_{k l}(x z)=\sigma_{i j}(x) \rho_{k l}(x) \chi(z)=\chi(z) \sigma_{i j} \rho_{k l}(x)$, so that $\sigma_{i j} \rho_{k l} \in \mathscr{F}(\chi)$. Moreover, as in the proof of Theorem (3.2) of [9], $\sigma_{i j} \rho_{k l}$ belongs to $\mathscr{F}_{r}(G)$, that is to $\mathscr{F}_{r}(\chi)$ (Proposition (1.1)).

Since each $f$ in $\mathscr{F}_{c}(G) \cap \mathscr{F}(\chi)$ can be approximated uniformly on $G$ by functions in $\mathscr{F}_{r}(\chi)$, i.e., by almost periodic functions, we have

COROLlaRY (1.3). $\mathscr{F}_{c}(G) \cap \mathscr{F}(\chi)$ is contained in the algebra of almost periodic functions on $G$. In particular these functions are uniformly continuous.

CoRollaRY (1.4). Let $G$ be a [Z]-group, $\chi$ an element of $Z^{\wedge}$, and $f$ in $\mathscr{F}_{z} \cap \mathscr{F}_{c}$ $\cap \mathscr{F}(\chi)$. Then $f$ can be approximated uniformly on $G$ by functions of the form $\sum_{k=1}^{n} c_{k} \chi_{\rho^{k}}$, where $c_{k} \in C$ and $\rho^{k} \in \lambda^{-1}(\chi)$, for $k=1, \ldots, n$.

Proof. Since $f \in \mathscr{F}_{c} \cap \mathscr{F}(\chi), f$ can be uniformly approximated on $G$ by some $r$ in $\mathscr{F}_{r}(\chi)$, and, in particular, on any compact set. As in the lemma of $\$ 1$ of [9], we have $G=U Z$ where $U$ is compact and invariant. Thus $\|f-r\|_{U}<\varepsilon$. We apply the \#-operator introduced in Theorem (1.1) and proceed as in the proof of Theorem (3.3) (both of [9]). Then

$$
(f-r)^{\#}=f^{\#}-r^{\#}=f-\sum_{k=1}^{n} c_{k} \chi_{\rho^{k}} .
$$

Moreover, $\left\|f-\sum c_{k} \chi_{\rho^{k}}\right\|_{U}=\left\|(f-r)^{\#}\right\|_{U} \leqq\|f-r\|_{U}<\varepsilon$. If $x=u z$ where $u \in U$ and $z \in Z$ then $f(x)=f(u) \chi(z)$ and $g(x)=g(u) \chi(z)$, where $g=\sum_{k=1}^{n} c_{k} \chi_{\rho}{ }^{k}$. Thus $|f(x)-g(x)|$ $=|f(u)-g(u)||\chi(z)|$, so that $\|f-g\|_{G}<\varepsilon$.

The next result is a refinement of Theorem (4.2) of [9] where the following is proven. Let $G$ be a $[Z]$-group and $f$ a nontrivial continuous complex function on $G$. Then $f / f(1)=\chi_{\rho} / \chi_{\rho}(1)$, for $\rho$ in $\mathscr{R}$, if and only if $f$ satisfies the character formula

$$
f(x) f(y)=f(1) \int_{G / Z} f\left(t x t^{-1} y\right) d t
$$

and is almost-periodic; $\rho$ is uniquely determined by $f$.

The merit of the following theorem derives from the fact that in applications we need not know beforehand that $f$ is almost-periodic. This will be significant in $\S 6$. 
THEOREM (1.5). Let $f$ be a nontrivial bounded continuous function on the [Z]-group $G$. Then $f$ satisfies the character formula if and only if $f / f(1)=\chi_{\rho} / \chi_{\rho}(1)$, for a unique $\rho$ in $\mathscr{R}(G)$.

Proof. By Theorem (4.2) of [9] it suffices to show that if $f$ is bounded and continuous and satisfies the character formula then $f$ is automatically almostperiodic. As in [9] we know $f(1) \neq 0$. By considering $f / f(1)$ we may assume that the character formula takes the form

$$
f(x) f(y)=\int_{G / Z} f\left(t x t^{-1} y\right) d t^{\circ} .
$$

It follows from (iv) that $f(z) f(y)=f(z y)$, for $z \in Z$ and $y \in G$. Hence the restriction $f_{Z}$ is a homomorphism of $Z$ into $C^{*}$, the multiplicative group of $C$. Thus $f(Z)$, and therefore also $f(Z)^{-}$, are subgroups of $C^{*}$. Since $f$ is bounded, $f(Z)^{-}$is a compact subgroup. However, the unit circle $T$ is the maximal compact subgroup, so that $f(Z)^{-} \subseteq T$. Since $f$ is continuous, we conclude that $f_{Z}=\chi \in Z^{\wedge}$. It follows that $f(z y)=\chi(z) f(y)$, for $z \in Z$ and $y \in G$. This means $f \in \mathscr{F}(\chi)$ and therefore, by Corollary (1.3), $f$ is almost-periodic.

2. The \#-operator and strong semisimplicity of $L_{1}(G)$. We now extend the \#-operator defined in $\S 1$ of [9] from $\mathscr{F}_{c_{0}}(G)$ to the $L_{p}$-spaces, $1 \leqq p<\infty$. Generalizing a definition made there we denote by $\mathscr{F}_{z}(G)$ the complex measurable functions $f$ on $G$ such that, for each $x$ in $G, f\left(x y x^{-1}\right)=f(y)$, almost everywhere in $y$ (where the null set depends on $x$ ). As is well known, for any unimodular group $G, \mathscr{F}_{z} \cap L_{1}$ $=\mathscr{Z}\left(L_{1}\right)$. This is deduced by use of the strong, and therefore weak, continuity of the left and right regular representations of $G$ on $L_{1}$. We also define ${ }^{\vee}: L_{p} \rightarrow L_{p}$, for $1 \leqq p \leqq \infty$, by $f^{\vee}(x)=f\left(x^{-1}\right)^{-}$. For $p=1$ this is standard notation, in view of the unimodularity of [Z]-groups [9, p. 366]. Moreover, $\left\|f^{\vee}\right\|_{p}=\|f\|_{p}$, for all $f$ in $L_{p}$ and $1 \leqq p \leqq \infty$. For $f$ in $L_{p}$, we write

$$
f^{\#}(y)=\int_{G / Z} f\left(x y x^{-1}\right) d x . \quad(y \in G, 1 \leqq p<\infty) .
$$

THEOREM (2.1). Let $G$ be $a[Z]$-group and $1 \leqq p<\infty$. Then \# is a well-defined linear operator on $L_{p}$, of norm 1 . Moreover, it is idempotent, commutes with ${ }^{`}$, preserves positive functions, and $L_{p}^{\#}=L_{p} \cap \mathscr{F}_{z}$.

In the case $p=1$, we have $L_{1}^{\#}=\mathscr{Z}\left(L_{1}\right)$ (by the above), $\left(f^{\#} * g\right)^{\#}=f^{\#} * g^{\#}\left(f, g \in L_{1}\right)$, and, in particular, $(f * g)^{\#}=f * g^{\#}\left(f \in \mathscr{Z}\left(L_{1}\right), g \in L_{1}\right)$. Finally $(f * g)^{\#}=(g * f)^{\#}$ $\left(f, g \in L_{1}\right)$. In the case $p=2$, \# is selfadjoint and therefore a projection.

If $f$ is a bounded uniformly continuous function and \# is defined as above then $f^{\#}$ is in $\mathscr{F}_{b} \cap \mathscr{F}_{u} \cap \mathscr{F}_{z}$ and one has $\left\|f^{\#}\right\|_{G} \leqq\|f\|_{G}$.

Proof. For $f$ in $L_{p}$, the function $\varphi: G / Z \times G \rightarrow C$ which sends $\left(x^{*}, y\right)$ into $f\left(x y x^{-1}\right)$, as well as $|\varphi|^{p}$, is measurable. Suppose $1<p<\infty$ and $1 / p+1 / q=1$. Then, since $1 \in L_{q}(G / Z)$, we have, by Hölder's Inequality, 


$$
\int_{G / Z}\left|f\left(x y x^{-1}\right)\right| 1\left(x^{*}\right) d x^{*} \leqq\left(\int_{G / Z}\left|f\left(x y x^{-1}\right)\right|^{p} d x^{*}\right)^{1 / p}\left(\int_{G / Z}\left|1\left(x^{*}\right)\right|^{q} d x^{\cdot}\right)^{1 / q} \cdot
$$

Since $G$ is unimodular, $\int_{G}\left|f\left(x y x^{-1}\right)\right|^{p} d y=\|f\|_{p}^{p}<\infty$. In view of the normalization of Haar measure on $G / Z$ this shows that $\int_{G / Z} \int_{G}\left|f\left(x y x^{-1}\right)\right|^{p} d y d x^{*}<\infty$. By the Fubini Theorem $\int_{G / Z}\left|f\left(x y x^{-1}\right)\right|^{p} d x$ exists a.e. in $y$ and

$$
\int_{G} \int_{G / Z}\left|f\left(x y x^{-1}\right)\right|^{p} d x^{\cdot} d y=\int_{G / Z} \int_{G}\left|f\left(x y x^{-1}\right)\right|^{p} d y d x=\|f\|_{p}^{p} .
$$

Hölder's Inequality then implies that $f^{\#}(y)$ exists a.e. Moreover, $\left|f^{\#}(y)\right|^{p} \leqq$ $\int_{G / Z}\left|f\left(x y x^{-1}\right)\right|^{p} d x^{\cdot}$ so that $\left\|f^{\#}\right\|_{p}^{p} \leqq\|f\|_{p}^{p}$. Thus $f^{\#} \in L_{p},\left\|f^{\#}\right\|_{p} \leqq\|f\|_{p}$.

If $p=1$ then, evidently, $\int_{G}\left|f^{\#}(y)\right| d y \leqq \int_{G} \int_{G / Z}\left|f\left(x y x^{-1}\right)\right| d x^{*} d y$. By the same calculation as that made above this equals $\|f\|_{1}$. Thus $f^{\#}$ is well defined in $L_{1}$ and $\left\|f^{\#}\right\|_{1} \leqq\|f\|_{1}$. If $f$ is in $\mathscr{F}_{b} \cap \mathscr{F}_{u}$ then $f^{\#}$ is in $\mathscr{F}_{u} \cap \mathscr{F}_{z}$ by Theorem (1.1) of [9]. Moreover,

$$
\operatorname{lub}_{y}\left|f^{\#}(y)\right| \leqq \operatorname{lub}_{y} \int_{G / Z}\left|f\left(x y x^{-1}\right)\right| d x \leqq\|f\|_{G} .
$$

Hence $\left\|f^{\#}\right\|_{G} \leqq\|f\|_{G}$ and $f^{\#} \in \mathscr{F}_{b}$. Clearly, \# is linear and preserves positive functions. It follows from the definition of \# that $f^{\#} \in \mathscr{F}_{z}(G)$. Thus \#: $L_{p} \rightarrow L_{p} \cap \mathscr{F}_{z}$. If $1 \leqq p<\infty$ and $f \in L_{p} \cap \mathscr{F}_{z}$, then $f=f^{\#}$ a.e.

Thus $L_{p}^{\#}=L_{p} \cap \mathscr{F}_{z}$ and \# is idempotent. It follows that the norm of \# is 1 . By unimodularity we have $\left(f^{\vee}\right)^{\#}(y)=\int_{G / Z} f\left(x y^{-1} x^{-1}\right)^{-} d x^{*}$, i.e., $f^{\#}\left(y^{-1}\right)^{-}=\left(f^{\#}\right)^{\vee}(y)$, so $\left(f^{\vee}\right)^{\#}=\left(f^{\#}\right)^{\vee}$.

If $p=1$, then

$$
(f * g)^{\#}(x)=\int_{G / Z} \int_{G} f\left(t x t^{-1} y\right) g\left(y^{-1}\right) d y d t^{\circ}
$$

Therefore

$$
\left(f^{\#} * g\right)^{\#}(x)=\int_{G / Z} \int_{G} \int_{G / Z} f\left(s t x t^{-1} y s^{-1}\right) d s^{\cdot} g\left(y^{-1}\right) d y d t .
$$

By the Fubini Theorem and the unimodularity of $G$ this is

$$
\int_{G} \int_{G / Z} \int_{G / Z} f\left(s t x t^{-1}\left(s^{-1} y s\right) s^{-1}\right) g\left(\left(s^{-1} y s\right)^{-1}\right) d s^{\cdot} d t^{\cdot} d y
$$

i.e.,

$$
\int_{G} \int_{G / Z} \int_{G / Z} f\left(s t x(s t)^{-1} y\right) g\left(s^{-1} y^{-1} s\right) d s^{\cdot} d t^{\cdot} d y .
$$

Applying the Fubini Theorem again and employing the map $y \rightarrow(s t) y(s t)^{-1}$ we obtain

$$
\int_{G} \int_{G / Z} \int_{G / Z} f\left(s t x y(s t)^{-1}\right) g\left(s^{-1}\left(s t y(s t)^{-1}\right)^{-1} s\right) d s d t d y
$$


i.e.,

$$
\int_{G} \int_{G / Z} \int_{G \mid Z} f\left(s t x y(s t)^{-1}\right) g\left(t y^{-1} t^{-1}\right) d s d t d y .
$$

Since the last expression equals

$$
\int_{G} \int_{G / Z} g\left(t y t^{-1}\right)\left(\int_{G / Z} f\left((s t) x y(s t)^{-1}\right) d s^{*}\right) d t^{\cdot} d y,
$$

where the inner integral is $f^{\#}(x y)$, we obtain

$$
\int_{G} \int_{G / Z} g\left(t y^{-1} t^{-1}\right) f^{\#}(x y) d t^{\cdot} d y=\int_{G} f^{\#}(x y) \int_{G / Z} g\left(t y^{-1} t^{-1}\right) d t^{\cdot} d y,
$$

i.e., $f^{\#} * g^{\#}(x)$. Thus $\left(f^{\#} * g\right)^{\#}=f^{\#} * g^{\#}$, for $f, g$ in $L_{1}$. Finally we show that $(f * g)^{\#}$ $=(g * f)^{\#}$, for $f, g$ in $L_{1}$. Using (i) we see that

$$
(g * f)^{\#}(x)=\int_{G / Z} \int_{G} g\left(t x t^{-1} y\right) f\left(y^{-1}\right) d y d t .
$$

This is

$$
\int_{G / Z} \int_{G} g(y) f\left(\left(\left(t x t^{-1}\right)^{-1} y\right)^{-1}\right) d y d t^{\circ} .
$$

By unimodularity this equals

$$
\left.\int_{G \mid Z} \int_{G} g\left(y^{-1}\right) f\left(\left(t x t^{-1}\right)^{-1} y^{-1}\right)^{-1}\right) d y d t^{\circ}
$$

which simplifies to

$$
\int_{G \mid z} \int_{G} g\left(y^{-1}\right) f\left(y t x t^{-1}\right) d y d t^{\circ}
$$

i.e.,

$$
\int_{G} g\left(y^{-1}\right)\left(\int_{G / Z} f\left(y t x t^{-1}\right) d t^{\cdot}\right) d y .
$$

Therefore, using (i), we see that it is sufficient to show

$$
\int_{G / Z} f\left(t x t^{-1} y\right) d t^{\cdot}=\int_{G / Z} f\left(y t x t^{-1}\right) d t^{*} .
$$

One establishes this by considering the map $t \rightarrow y^{-1} t$.

If $p=2$, then $\left\langle f^{\#}, g\right\rangle=\int_{G} \int_{G / Z} f\left(x y x^{-1}\right) g(y)^{-1} d x^{*} d y$. On the other hand, $\left\langle g^{\#}, f\right\rangle^{-}=\int_{G} \int_{G \mid Z} g\left(x y x^{-1}\right)^{-} f(y) d x^{*} d y$. One sees that \# is selfadjoint by applying unimodularity twice.

THEOREM (2.2). Let $G$ be $a[Z]$-group and I be any closed 2-sided ideal in $L_{1}(G)$. Then $I^{\#} \subset I$; in fact, $I^{\#}=I \cap \mathscr{Z}\left(L_{1}\right)$. In particular, the strong radical of $L_{1}(G)$ is \#-stable. 
Proof. Let $f \in I$. Then, since $I$ is a left ideal, $f_{x} \in I$, for each $x$ in $G$. Using the fact that $I$ is also a right ideal we see that $\left(f_{x}\right)^{x-1}=x \Delta f \in I$, for all $x$ in $G$. Consider the pairing $L_{1} \times L_{\infty} \rightarrow C$ given by

$$
(g, \psi) \rightarrow \int_{G} g(x) \psi(x) d x \quad\left(g \in L_{1}, \psi \in L_{\infty}\right)
$$

Suppose $\psi \in I^{\perp}$ (orthogonal complement). Then

$$
\int_{G} f^{\#}(y) \psi(y) d y=\int_{G}\left(\int_{G} w(x) f\left(x y x^{-1}\right) d x\right) \psi(y) d y .
$$

This equals $\int_{G} w(x)\left(\int_{G} f\left(x y x^{-1}\right) \psi(y) d y\right) d x=\int w(x)(x \Delta f, \psi) d x$. Since $x \Delta f \in I$, for all $x$, and $\psi \in I^{\perp},(x \triangle f, \psi)=0$ on $G$, and therefore $\left(f^{\#}, \psi\right)=0$. Hence $f^{\#} \in I^{\perp \perp}$. Since $L_{1}^{*}=L_{\infty}$ and $I$ is closed it follows from $[16,8 C]$ that $I^{\perp \perp}=I$. Thus $f^{\#} \in I$ and $I^{\#} \subseteq I$. Then evidently $I^{\#}=I \cap \mathscr{Z}\left(L_{1}\right)$.

The next lemma is used in the proof of Theorem (2.4) as well as (6.11).

Lemma (2.3). If $G$ is an [SIN]-group then $\mathscr{F}_{u} \cap L_{1}$ is a dense 2-sided ideal in $L_{1}$. In particular, if $f$ belongs to $\mathscr{F}_{u} \cap L_{1}$ then so does $f * f^{\vee}$.

Proof. The density follows from the fact that $\mathscr{F}_{c_{0}}$ is dense in $L_{1}$. Let $f \in \mathscr{F}_{u} \cap L_{1}$ and $g \in L_{1}$. Then we show $f * g \in \mathscr{F}_{u}$. For $x_{1}, x_{2} \in G$, we have

$$
\left|f * g\left(x_{1}\right)-f * g\left(x_{2}\right)\right| \leqq \int_{G}\left|f\left(x_{1} y\right)-f\left(x_{2} y\right)\right|\left|g\left(y^{-1}\right)\right| d y .
$$

By uniform continuity of $f$ and the fact that $G \in[S I N]$ it follows that

$$
\left|f\left(x_{1} y\right)-f\left(x_{2} y\right)\right|<\varepsilon
$$

for all $y$ in $G$, if $x_{1}^{-1} x_{2}$ is in some neighborhood of 1 in $G$. By unimodularity, we then have $\left|f * g\left(x_{1}\right)-f * g\left(x_{2}\right)\right| \leqq \varepsilon\|g\|_{1}$. A similar argument shows $g * f \in \mathscr{F}_{u}$.

The following theorem, as announced in the introduction, generalizes a result of Segal [22], while making use of essentially his method of proof. In the next section we shall give a further generalization as well as a sharpening of it via a different method.

THEOREM (2.4). If $G$ is a [Z]-group then $L_{1}(G)$ is strongly semisimple.

Proof. Let $g \in \mathscr{F}_{c_{0}}$ and $f \in$ st. rad. $\left(L_{1}\right)$ (strong radical). Denote $(f * g) *(f * g)^{\vee}$ by $\varphi$. Since st. rad. $\left(L_{1}\right)$ is a 2 -sided (and therefore left) ideal, it contains $\varphi$. It follows that $\varphi^{\#} \in$ st. $\operatorname{rad} .\left(L_{1}\right) \cap \mathscr{Z}\left(L_{1}\right)$. By Theorem (1.5) of [22] (which applies to any locally compact group) we have $\varphi^{\#}=0$ a.e. On the other hand, since $f \in L_{1}$, and $g \in \mathscr{F}_{c_{0}} \subseteq L_{\infty}$, we know that $f * g$ belongs to $\mathscr{F}_{u} \cap L_{1}$; but then so does $\varphi$, by Lemma (2.3). It follows from Theorem (1.1) of [9] that $\varphi^{\#} \in \mathscr{F}_{u}$ and therefore $\varphi^{\#}=0$ on $G$. In particular,

$$
\varphi^{\#}(1)=\int_{G / Z} \varphi(1) d t^{\cdot}=\varphi(1)=\|f * g\|_{2}^{2}=0 .
$$


Hence $f * g=0$ a.e., so that $\|f * g\|_{1}=0$. Now choose an approximate identity $\left\{g_{v}\right\}$ from $\mathscr{F}_{c_{0}}$. Then $\left\|f-f * g_{v}\right\|_{1} \rightarrow 0$. The triangle inequality implies that $\|f\|_{1}=0$.

3. A characterization of $[M A P]$-groups and uniqueness theorems for Fourier transforms. In $\S 2$ we showed that the group algebra $L_{1}(G)$ of a $[Z]$-group is strongly semisimple. The definition given below introduces a stronger type of semisimplicity in terms of a new radical.

Definition. If $A$ is a Banach algebra, fin. $\operatorname{rad} .(A)$ is the intersection of all regular maximal 2-sided ideals of finite codimension. (In the case of a *-algebra one can use *-ideals for the definition of the finite radical.) We shall call $A$ finitely semisimple if fin. $\operatorname{rad} .(A)=(0)$.

THEOREM (3.1). Let $G$ be a locally compact group. Then its group algebra $L_{1}(G)$ is finitely semisimple if and only if $G$ is an [MAP]-group.

The proof of Theorem (3.1) will be given presently. We first recall some facts about the Fourier transform. Let $\rho$ be a continuous unitary representation of the locally compact group $G$ on the Hilbert space $V$. Then $T_{f}(\rho)=\int f(x) \rho(x) d x$ (operator-valued integral) defines a bounded operator on $V$, for each $f \in L_{1}$, which is characterized by $\left\langle T_{f}(\rho)(v), w\right\rangle=\int_{G} f(x)\left\langle\rho_{x}(v), w\right\rangle d x$ for $v, w \in V$. In fact, $\left\|T_{f}(\rho)\right\| \leqq\|f\|_{1}$, and $T(\rho)$ is a nondegenerate continuous *-representation of the *-algebra $L_{1}$ on $V$. Moreover, one has $T_{f_{x-1}}(\rho)=\rho(x) T_{f}(\rho)$, for $x \in G, f \in L_{1}$. Conversely, to each nondegenerate continuous *-representation $L$ corresponds a unique $\rho$ as above whose Fourier transform $T(\rho)=L$. The commuting algebras of $\rho$ and $T(\rho)$ are equal so that irreducibility and equivalence occur simultaneously for $\rho$ and $T(\rho)$. We shall also have occasion to define $T_{\mu}(\rho)$ where $\mu$ is a finite regular Borel measure on $G$ (not necessarily positive). This is done in a similar way, i.e., $T_{\mu}(\rho)=\int \rho(x) d \mu$, and gives rise to a representation of the measure algebra $M(G)$. If $\mu$ is absolutely continuous with respect to Haar measure then this evidently coincides with the Fourier transform on $L_{1}$. For a full discussion of the foregoing see [13] and [16].

In the case $\rho \in \mathscr{R}_{\mathrm{fn}}(G)$ and $G$ is an [MAP]-group, by taking the trace, we define another type of Fourier transform which will be especially useful when considering central functions; namely, $f^{\wedge}(\rho)=\operatorname{tr}\left(T_{f}(\rho)\right)$. As we shall see, the relation $\left(f_{x^{-1}}\right)^{\wedge}(\rho)$ $=d_{\rho}^{-1} \chi_{\rho}(x) f^{\wedge}(\rho)$, for $f \in \mathscr{Z}\left(L_{1}\right)$ and $x \in G$, follows from the above together with the proof of Corollary (3.3).

As is known, one has, for an arbitrary locally compact group $G$, a uniqueness theorem for the Fourier transform: If $f$ and $g \in L_{1}$ and $T_{f}(\rho)=T_{g}(\rho)$ for all $\rho \in \mathscr{R}(G)$, then $f=g$ a.e. More generally, if $\mu, \nu \in M(G)$ and $T_{\mu}(\rho)=T_{v}(\rho)$, for all $\rho \in \mathscr{R}$, then $\mu=\nu$. In the case of an [MAP]-group one can sharpen this result as follows.

Theorem (3.2) (Uniqueness TheOrem for the Fourier TRANSFORM). Let $G$ be an $[M A P]$-group and $\mu, \nu \in M(G)$. If $T_{\mu}(\rho)=T_{\nu}(\rho)$, for all $\rho \in \mathscr{R}_{\mathrm{fn}}(G)$, then $\mu=\nu$. In particular, this holds for functions in $L_{1}$. 
Proof. Since $\mu-\nu \in M(G)$ and $T(\rho)$ is linear we may assume that $\nu=0$, i.e., that $\int \rho(x) d \mu(x)=0$, for all $\rho \in \mathscr{R}_{\text {fin }}$, and show $\mu=0$. We first do this in the case $G$ compact as follows $\left({ }^{3}\right)$. The linearity of $\int_{G} d \mu$ shows that,

$$
\int_{G} r(x) d \mu(x)=0
$$

for all $r \in \mathscr{F}_{r}$, the algebra of representative functions associated with $\rho \in \mathscr{R}_{\text {fin }}$. By the Peter-Weyl Theorem this implies that (i) holds for all $f \in \mathscr{F}_{c}$. If we write $d \mu=h d|\mu|$ where $\|h\|_{\infty}=1$ and $d|\mu|$ is a positive regular measure (see W. Rudin, Real and complex analysis, McGraw-Hill, New York, 1966, p. 126) then, since $\mathscr{F}_{c}$ is dense in $L_{1}(|\mu|)$, we have $\int f h d|\mu|=0$, for all $f \in L_{1}(|\mu|)$. This shows that $\|h\|_{\infty}=0$ or else $|\mu|=0$. Thus $\mu=0$.

Returning to the general case we have $\mu \in M(G)$ and assume that $\int_{G} \rho(x) d \mu(x)=0$, for all $\rho \in \mathscr{R}_{\text {fln }}$. As above this implies that (i) holds for all $r \in \mathscr{F}_{r}$. Let $\alpha: G \rightarrow G^{\alpha}$ be the imbedding of $G$ into its Bohr group. For $\varphi \in \mathscr{F}_{c}\left(G^{\alpha}\right)$, define $L(\varphi)=\int_{G} \varphi \alpha d \mu$. Since $\varphi \alpha$ is continuous on $G$ it is $d \mu$-integrable. Moreover, since $|L(\varphi)| \leqq\|\mu\|\|\varphi\|_{G^{\alpha}}$, for all $\varphi, L$ is a bounded linear functional on $\mathscr{F}_{c}\left(G^{\alpha}\right)$. By the Riesz theorem, there exists a $\mu^{\alpha} \in M\left(G^{\alpha}\right)$ such that

$$
\int_{G^{\alpha}} \varphi d \mu^{\alpha}=\int_{G} \varphi \alpha d \mu,
$$

and so that $\|L\|=\left\|\mu^{\alpha}\right\| \leqq\|\mu\|$. By the regularity of $d\left|\mu^{\alpha}\right|, \mathscr{F}_{c}\left(G^{\alpha}\right)$ is dense in $L_{1}\left(\left|\mu^{\alpha}\right|\right)$, and therefore (ii) holds for all $\varphi \in L_{1}\left(\left|\mu^{\alpha}\right|\right)$ and in particular for $\varphi_{B}$, the characteristic function of a Borel set $B \subseteq G^{\alpha}$. Now, since $\varphi_{B} \alpha=\varphi_{\alpha^{-1}(B)}$, (ii) shows that

$$
\mu^{\alpha}(B)=\mu\left(\alpha^{-1}(B)\right) \text {. }
$$

Here $\alpha^{-1}(B)$ is a Borel set in $G$ since $\alpha$ is continuous. Now let $r^{\alpha} \in \mathscr{F}_{r}\left(G^{\alpha}\right)$; then there exists a unique $r \in \mathscr{F}_{r}(G)$ satisfying $r=r^{\alpha} \alpha$. Thus $\int_{G^{\alpha}} r^{\alpha} d \mu^{\alpha}=\int_{G} r^{\alpha} \alpha d \mu=\int_{G} r d \mu=0$. Since $G^{\alpha}$ is compact this means $\mu^{\alpha}=0$. It follows from (iii) that $\mu\left(\alpha^{-1}(B)\right)=0$, for all Borel sets $B \subseteq G^{\alpha}$. Let $F$ be a compact set in $G$; then $\alpha(F)$ is compact and hence a Borel set. This means that $\mu\left(\alpha^{-1}(\alpha(F))\right)=\mu(F)=0$, because $\alpha$ is injective. By regularity we find that $\mu=0$.

Proof of Theorem (3.1). Let $G$ be an [MAP]-group and $\rho \in \mathscr{R}_{\mathrm{fn}}$. Then the Fourier transform $T(\rho): L_{1} \rightarrow M_{d_{\rho}}(C)$ is a finite-dimensional irreducible *-representation of $L_{1}$. Since $T(\rho)$ is nondegenerate, it is nontrivial. Thus $T(\rho)\left(L_{1}\right)$ is a nontrivial irreducible subalgebra of the matrix algebra $M_{d_{\rho}}(C)$. By Burnside's Theorem (N. Jacobson, Lectures in abstract algebra. II. Linear algebra, Van Nostrand, Princeton, N. J., 1952, p. 276), $T(\rho)$ is surjective. It follows that

$$
L_{1} / \operatorname{Ker} T(\rho) \cong M_{d_{\rho}}(C) .
$$

$\left({ }^{3}\right)$ In the case of a compact group the uniqueness also follows from the general result quoted above together with the fact that $\mathscr{R}$ coincides with $\mathscr{R}_{\mathbf{f i n}}$. Similarly, uniqueness also follows directly for [ $Z$ ]-groups via Theorem (2.1) of [9]. 
Since $M_{d_{\rho}}(C)$ is a simple algebra with identity, $\operatorname{Ker} T(\rho)$ is a regular maximal 2-sided ideal whose codimension is evidently $d_{\rho}^{2}$. Since $T(\rho)$ is a *-homomorphism, this ideal is even ${ }^{*}$-stable. We complete the proof by observing that

$$
\bigcap\left\{\operatorname{Ker} T(\rho) \mid \rho \in \mathscr{R}_{\mathrm{fn}}\right\}=(0),
$$

by Theorem (3.2).

Conversely, let $G$ be a locally compact group and suppose fin. $\operatorname{rad} .\left(L_{1}\right)=(0)$ (where, for the moment, we do not regard $L_{1}$ as a *-algebra). Let $\rho$ denote the left regular representation of $G$ on $L_{1}$ and $I$ a closed left ideal. Since $I$ is a $\rho$-stable subspace this induces an action $x \rightarrow\left(\rho_{I}\right)_{x}$ of $G$ on $L_{1} / I$. This representation is uniformly bounded and strongly continuous. In particular, if $I$ is a regular maximal 2-sided ideal of finite codimension the representation $x \rightarrow\left(\rho_{I}\right)_{x}$ is a finite-dimensional uniformly bounded representation of $G$. If $x \in G$ and $\left(\rho_{I}\right)_{x}=$ identity, for each such $I$, then, for $f \in L_{1}$, we have $\left(\rho_{I}\right)_{x}(f+I)=f+I=f_{x}+I$. Thus $f-f_{x} \in I$, for all such $I$; i.e., $f-f_{x} \in$ fin. $\operatorname{rad} .\left(L_{1}\right)=(0)$. Since $f$ is arbitrary and $\rho$ is faithful, $x=1$; thus the finite-dimensional continuous uniformly bounded representations $\rho_{I}$ of $G$ separate the points. Let $I$ be fixed and consider $\rho_{I}(G)$. This is a bounded subgroup of $\mathrm{Gl}(n, \boldsymbol{C})$ where $n=$ codimension of $I$, and hence its closure is a compact subgroup. It can therefore be conjugated into $U_{n}(\boldsymbol{C})$, a maximal compact subgroup of $\mathrm{Gl}(n, C)$. This means that $\rho_{I}$ is boundedly equivalent to a finite-dimensional continuous unitary representation $\sigma_{I}$ of $G$, for each $I$. If $\sigma_{I}=$ identity, for $I$, then $\left(\sigma_{I}\right)_{x}=B_{I}\left(\rho_{I}\right)_{x} B_{I}^{-1}$ for $x \in G$, so that $\left(\rho_{I}\right)_{x}=$ id, for all $I$, and therefore $x=1$. Thus the finite-dimensional continuous unitary representations separate the points of $G$ and therefore, by semisimplicity, so do their irreducible components.

As a corollary of the above results we get a uniqueness theorem for the Fourier transform of a central function.

Corollary (3.3). Let $G$ be an [MAP]-group and $f, g \in \mathscr{Z}\left(L_{1}(G)\right)$. If $f^{\wedge}(\rho)=g^{\wedge}(\rho)$, for all $\rho \in \mathscr{R}_{\mathrm{fn}}(G)$, then $f=g$ a.e.

Proof. By linearity we may evidently assume $g=0$. As was shown above, $T(\rho): L_{1} \rightarrow M_{d_{\rho}}(C)$ is surjective. This implies that since $T(\rho)$ is an algebra homomorphism, $T(\rho)\left(\mathscr{Z}\left(L_{1}\right)\right) \subseteq \mathscr{Z}\left(M_{d_{\rho}}(C)\right)$. Because the latter consists of scalar multiples of the identity, $T_{f}(\rho)=\lambda_{\rho} I_{d_{\rho}}$, so that $f^{\wedge}(\rho)=\lambda_{\rho} d_{\rho}$. This means $\lambda_{\rho}=0$ and therefore $T_{f}(\rho)=0$, for all $\rho$. The result now follows from Theorem (3.2).

We conclude $\$ 3$ with two examples. Example (3.4) illustrates the fact that a group may have a finitely semisimple group algebra but have infinite-dimensional irreducible unitary representations and Example (3.5) shows that, in general, the finite radical is larger than the strong radical.

EXAMPLE (3.4). Let $G$ be the discretely topologized free group on finitely many generators. As is well known, $G$ is residually finite [Kurosh, Group theory, vol. II] and therefore in $[M A P]$. Hence, by (3.1), it has finite radical (0). On the other hand it must have infinite-dimensional irreducible unitary representations; for if 
not, then $G$ would be of type I. A theorem of Thoma [26] would then show that since $G$ is countable and discrete, it has a normal abelian subgroup of finite index, an evident impossibility for a free group.

EXAMPLE (3.5). Let $G$ be the group defined in Example (5.10) of [11]. There we showed that $G$ is not an $[M A P]$-group. Hence, by $(3.1), \operatorname{fin} . \operatorname{rad} .\left(L_{1}(G)\right) \neq(0)$. On the other hand, since $G$ is a discrete $[F C]$-group, $L_{1}(G)$ is strongly semisimple $\left({ }^{4}\right)$.

4. The Plancherel Formula for $[Z]$-groups. Since the canonical map $\lambda: \mathscr{R}(G)$ $\rightarrow Z^{\wedge}$ is surjective this gives a partition of $\mathscr{R}(G)$ into the disjoint union of nonempty sets $\lambda^{-1}(\chi)$, for $\chi \in Z^{\wedge} . \mathscr{R}(G)$ is the space on which the Plancherel measure $\mu_{\mathrm{pl}}$ lives.

Theorem (4.1) (The Plancherel Theorem). Let $G$ be $a[Z]$-group and $f, g \in L_{1}$ $\cap L_{2}(G)$. Then

$$
\langle f, g\rangle=\int_{Z^{\wedge}} \sum_{\rho \in \lambda^{-1}(x)} d_{\rho} \operatorname{tr}\left(T_{f}(\rho) T_{g}(\rho)^{*}\right) d \chi,
$$

where $\int_{Z^{\wedge}} d \chi$ is Haar measure on $Z^{\wedge}$. That is, for each $\chi$ in $Z^{\wedge}$, except for a set of measure 0 , the function $\rho \rightarrow d_{\rho} \operatorname{tr}\left(T_{f}(\rho) T_{g}(\rho)^{*}\right)$ defined on $\lambda^{-1}(\chi)$ is 0 except for countable many $\rho$ 's and is absolutely summable. The resulting function on $Z^{\wedge}$ is in $L_{1}\left(Z^{\wedge}\right)$ and its integral equals $\langle f, g\rangle$.

Thus the Plancherel measure is defined as follows. A subset $E$ of $\mathscr{R}(G)$ is summable if the function $\chi \rightarrow$ card $\left(E \cap \lambda^{-1}(\chi)\right)$ is in $L_{1}\left(Z^{\wedge}\right)$; and then

$$
\mu_{\mathrm{pl}}(E)=\int_{\mathrm{z}^{\wedge}} \operatorname{card}\left(E \cap \lambda^{-1}(\chi)\right) d \chi
$$

As will be shown, the result generalizes and unifies in a natural manner the classical Plancherel Theorems for compact and abelian groups (see Naìmark [21, p. 436 and 414]). Although, in a sense, there are more general results known, namely for [SIN]-groups (Godement [6]), or even for arbitrary unimodular groups (Sunouchi [25]), as well as (Segal [23]) for locally compact separable unimodular groups, the first two results quoted above are given in the following form: If $f, g \in L_{1} \cap L_{2}$, then

$$
\langle f, g\rangle=\int_{\mathscr{R}(G)} \operatorname{tr}\left(T_{f}(\rho) T_{g}(\rho)^{*}\right) d \mu(\rho),
$$

for some unique measure $\mu$ on $\mathscr{R}(G)$ (or a set in bijective correspondence with $\mathscr{R}(G)$ ), where tr is a generalized trace appropriate for infinite-dimensional representations. The measure $\mu$ is called the Plancherel measure. As remarked above, the virtue of our result is not that of great generality but rather that it gives an explicit description of the Plancherel measure in the case of a [Z]-group. Here,

$\left.{ }^{4}\right)$ This fact was recently proven by R. Mosak and will appear in his doctoral dissertation (Columbia University). 
$\mathscr{R}(G)$ consists of only finite-dimensional representations and the trace is the usual one. At the moment the explicit description seems to be important in Corollaries (4.5) and (4.6) below, although the former result probably holds in greater generality. Also in [25] and [23] the unity with the classical compact and abelian theory seems to be largely lost. Our methods are very much in the spirit of, and depend on, compact and abelian group theory. They do not explicitly use the notion of direct integral, square summable vector field etc. and therefore can be considered more elementary than the above. Another Plancherel Theorem, for CCR-groups, due to Kaplansky (proof unpublished) is quoted in [17, p. 405]. Its form and scope is analogous to that of [6], for the case of [SIN]-groups. As above, because of the generality achieved, the measure is not given explicitly.

A word about normalizations seems appropriate here. The Haar measures on $G, Z, G / Z$ are arbitrary except for normalizations $\int_{G / Z}=1$ and $\int_{G}=\int_{G / Z} \int_{Z}$, thus giving one free parameter, $\int_{Z}$ say. Since $\int_{Z^{\wedge}}$ is determined by $\int_{Z}$ and the abelian Plancherel formula, $\mu_{\mathrm{pl}}$ is also determined only up to a constant. Of course, if one did not want the factor $d_{\rho}$ to appear in the formula this could be absorbed by not using counting measure. The formula would then be of the type mentioned above.

Before giving the proof of (4.1) we require some preliminaries. In what follows $G$ will always denote a $[Z]$-group and $\chi$ a fixed character in $Z^{\wedge}$.

DEFINITION (4.2). If $f, g \in \mathscr{F}(\chi)$ then, clearly, $f g^{-}$is a measurable function on $G / Z$. Let $\mathscr{F}(\chi)_{2}=\left\{\left.f\left|f \in \mathscr{F}(\chi), \int_{G / Z}\right| f\left(x^{*}\right)\right|^{2} d x^{\circ}<\infty\right\}$. Then $\mathscr{F}(\chi)_{2}$ is a linear subspace of $\mathscr{F}(\chi)$. For $f, g \in \mathscr{F}(\chi)_{2}$, we write $\langle f, g\rangle_{x}=\int_{G / Z} f g^{-} d x^{\cdot}$ (which exists). With this definition of inner product and suitable identification, $\mathscr{F}(\chi)_{2}$ becomes a complex Hilbert space. We denote its norm by $\|f\|_{x}$.

If $f \in \mathscr{F}_{c}(G) \cap \mathscr{F}(\chi)$ then $|f|^{2}$ is a continuous function on the compact group $G / Z$ and therefore $\|f\|_{\chi}^{2}<\infty$, so that $\mathscr{F}_{c}(G) \cap \mathscr{F}(\chi) \subseteq \mathscr{F}(\chi)_{2}$. In fact,

$$
\|f\|_{x}^{2}=\int_{G / Z}\left|f\left(x^{\cdot}\right)\right|^{2} d x^{\cdot}=\int_{G} w(x)|f(x)|^{2} d x,
$$

by Theorem (1.2) of [9]. The latter is $\leqq\|f\|_{\operatorname{supp} w}^{2} \int_{G} w(x) d x \leqq\|f\|_{G}^{2}$ since this last integral is 1 . Thus $\|f\|_{x} \leqq\|f\|_{G}<\infty$, for all $f \in \mathscr{F}_{c}(G) \cap \mathscr{F}(\chi)$.

Next we define a Fourier transform with respect to $\chi$. Let $f \in L_{1} \cap L_{2}(G)$; then $P_{x}(f)(x)=\left(f_{x}\right)^{\wedge}(\chi)=\int_{z} f_{x}(z) \chi(z)^{-} d z, x \in G$. Now $P_{\chi}(f) \in \mathscr{F}(\chi)$, for all $f \in L_{1} \cap L_{2}$. For $z_{1} \in Z$, we have

$$
\begin{aligned}
P_{\chi}(f)\left(x z_{1}\right) & =\int_{Z} f_{x z_{1}}(z) \chi(z)^{-} d z=\int_{z} f_{x}\left(z_{1} z\right) \chi(z)^{-} d z \\
& =\int_{z} f_{x}(z) \chi\left(z_{1}^{-1} z\right)^{-} d z=\chi\left(z_{1}\right) P_{\chi}(f)(x) .
\end{aligned}
$$

Thus we have a linear operator $P_{\chi}: L_{1} \cap L_{2} \rightarrow \mathscr{F}(\chi)$. We show that $P_{\chi}\left(\mathscr{F}_{c_{0}}(G)\right)$ is contained in $\mathscr{F}(\chi) \cap \mathscr{F}_{c}(G)$ and, in particular, in $\mathscr{F}(\chi)_{2}$. To see this, let $x_{0} \in G$, $U_{*}$ be a fixed compact neighborhood of 1 , and $\varepsilon>0$. Choose a symmetric neigh- 
borhood $U \subseteq U_{*}$ of 1 satisfying $|f(x)-f(y)|<\varepsilon$ if $x y^{-1} \in U$. Let $x x_{0}^{-1} \in U$ and $z \in Z$. Then $(x z)\left(x_{0} z\right)^{-1} \in U$, so that $\left|f(x z)-f\left(x_{0} z\right)\right|<\varepsilon$ for all $z \in Z$. Since $\left|f_{x}-f_{x_{0}}\right|$ $\leqq\left|f_{x}\right|+\left|f_{x_{0}}\right|$ and $\operatorname{supp} f_{t}=t^{-1} \operatorname{supp} f$, for all $t \in G$, we have $\operatorname{supp}\left|f_{x}-f_{x_{0}}\right| z \subseteq$ $\left(x^{-1} \operatorname{supp} f \cup x_{0}^{-1} \operatorname{supp} f\right) \cap Z$. On the other hand, $U$ is symmetric, so $x^{-1} \in x_{0}^{-1} U$ and therefore $x^{-1} \operatorname{supp} f \subseteq x_{0}^{-1} U \operatorname{supp} f \subseteq x_{0}^{-1} U_{*} \operatorname{supp} f$. Hence $\operatorname{supp}\left|f_{x}-f_{x_{0}}\right|_{z}$ $\subseteq\left(x_{0}^{-1} U_{*} \operatorname{supp} f\right) \cap Z$, a fixed compact set $K$ in $Z$. Now

$$
\left|P_{x}(f)(x)-P_{\chi}(f)\left(x_{0}\right)\right| \leqq \int_{z}\left|f_{x}(z)-f_{x_{0}}(z)\right| d z \leqq \varepsilon \mu_{z}(K)
$$

if $x x_{0}^{-1} \in U$. Thus $P_{x}(f)$ is continuous on $G$.

Let $f \in \mathscr{F}_{c_{0}}(G)$ and $g \in \mathscr{F}(\chi)_{2}$. Then (see $[16$, p. 131])

$$
\begin{aligned}
\int_{G} f(x) g(x)^{-} d x & =\int_{G / Z} \int_{Z} f(x z) g(x z)^{-} d z d x^{*}=\int_{G / Z} g\left(x^{*}\right)^{-}\left(\int_{Z} f(x z) \chi(z)^{-} d z\right) d x^{\cdot} \\
& =\left\langle P_{\chi}(f), g\right\rangle_{x} .
\end{aligned}
$$

Now suppose $\left\langle P_{\chi}(f), g\right\rangle_{x}=0$ for all $f \in \mathscr{F}_{c_{0}}(G)$ and fixed $g \in \mathscr{F}(\chi)_{2}$. Then we have

(i) $\int_{G} f(x) g(x)^{-} d x=0$ and

(ii) $\int_{G / Z}\left|g\left(x^{*}\right)\right|^{2} d x^{*}=\int_{G} w(x)|g(x)|^{2} d x<\infty$.

Let $d \nu=w d x$. Then $d v$ is a regular positive Borel measure on $G$ and therefore $\mathscr{F}_{c_{0}}(G)$ is dense in $L_{2}(\nu)$. Equation (ii) says that $g \in L_{2}(v)$. If $f$ is in $\mathscr{F}_{c_{0}}(G)$ then so is $w f$, so that (i) shows $\int w(x) f(x) g(x)^{-} d x=0$, i.e., $\langle f, g\rangle=0$ in $L_{2}(v)$, for all $f \in \mathscr{F}_{c_{0}}(G)$. It follows that $g=0$ a.e. in $\nu$, i.e. $\int_{G} w(x)|g(x)|^{2} d x=\|g\|_{x}^{2}=0$. Since $P_{x}\left(\mathscr{F}_{c_{0}}(G)\right)$ is a linear subspace of $\mathscr{F}(\chi)_{2}$ this implies that it, and hence also $\mathscr{F}(\chi) \cap \mathscr{F}_{c}(G)$, is dense in $\mathscr{F}(\chi)_{2}$.

In light of the foregoing discussion we may reinterpret our previous results as follows:

THEOREM (4.2). The set $\left\{d_{\rho}^{1 / 2} \rho_{i j} \mid i, j=1, \ldots, d_{\rho}, \rho \in \lambda^{-1}(\chi)\right\}$ is an orthonormal basis of $\mathscr{F}(\chi)_{2}$.

Proof. As was proven in [9], these functions are in $\mathscr{F}(\chi)_{2}$ and are orthonormal. By the above, if $f \in \mathscr{F}(\chi)_{2}$, there exists a $g \in \mathscr{F}(\chi) \cap \mathscr{F}_{c}(G)$ such that $\|f-g\|_{x}<\varepsilon$. By Theorem (1.2), we can find $r \in \mathscr{F}_{r}(\chi)$ so that $\|g-r\|_{G}<\varepsilon$. Hence $\|g-r\|_{\chi} \leqq$ $\|g-r\|_{G}<\varepsilon$ and therefore $\|f-r\|_{x}<2 \varepsilon$.

REMARK. It follows from the above that $\chi^{G}$, the induced representation of $\chi \in Z^{\wedge}$ to $G$, decomposes into $\sum_{\rho \in \lambda^{-1}(x)} \oplus d_{\rho} \rho$ on $\mathscr{F}(\chi)_{2}$. That is, $\chi^{G}$ is the orthogonal direct sum of finite-dimensional continuous irreducible unitary representations of $G$. Each $\rho$ occurs (up to equivalence) $d_{\rho}$ times if $\rho \in \lambda^{-1}(\chi)$, and otherwise does not occur. (In the case of separable $G$, this follows from a Frobenius Reciprocity Theorem of Mackey [19].) To see this, note that for $\rho \in \lambda^{-1}(\chi)$ the space $R(\rho)$ spanned by $\left\{\rho_{i j} \mid i, j=1, \ldots, d_{\rho}\right\}$ is a $d_{\rho}^{2}$-dimensional, translation (i.e. $\chi^{G}$ )-invariant subspace of $\mathscr{F}(\chi)_{2}$ and that $\mathscr{F}(\chi)_{2}$ is the orthogonal direct sum of $\left\{R(\rho) \mid \rho \in \lambda^{-1}(\chi)\right\}$. 
It is easy to see that $\operatorname{tr}\left(\left(\chi^{G}\right)_{R(\rho)}\right)(x)=d_{\rho} \chi_{\rho}(x)$, for $x \in G$. Since $\left(\chi^{G}\right)_{R(\rho)}$ is a finitedimensional unitary representation, its semisimplicity yields

$$
\left(\chi^{G}\right)_{R(\rho)}(x)=\sum_{i=1}^{r} \oplus n_{i} \sigma_{i}(x)
$$

where the $\sigma_{i}$ are irreducible and unequivalent and each $\sigma_{i}$ occurs with multiplicity $n_{i}$. Hence $\sum_{i=1}^{r} n_{i} \chi_{\sigma_{i}}(x)=d_{\rho} \chi_{\rho}(x)$. It follows from the theorem of Frobenius and Schur [9] that $r=1, \sigma_{1}=\rho$ and $n_{1}=d_{\rho}$. Thus $\chi^{G}=\sum_{\rho \in \lambda^{-1}(x)} \oplus d_{\rho} \rho$.

Before stating the next result we define a \#-operator on $\mathscr{F}(\chi)_{2}$ as follows: If $f \in \mathscr{F}(\chi)_{2}$ then $f^{\#}(x)=\int_{G / Z} f\left(y x y^{-1}\right) d y^{\circ}$, for $x$ in $G$. If $z \in Z$, we have

$$
\begin{aligned}
f^{\#}(z x) & =\int_{G / Z} f\left(y z x y^{-1}\right) d y^{\circ}=\int_{G / Z} f\left(y x y^{-1} z\right) d y^{\cdot}=\int_{G / Z} f\left(y x y^{-1}\right) \chi(z) d y^{\circ} \\
& =\chi(z) f^{\#}(x) .
\end{aligned}
$$

Moreover,

$$
\begin{aligned}
\left\|f^{\#}\right\|_{x} & =\left(\int_{G / Z}\left|f^{\#}\left(x^{*}\right)\right|^{2} d x^{\cdot}\right)^{1 / 2}=\left[\int_{G / Z}\left|\int_{G / Z} f\left(y x y^{-1}\right) d y^{\cdot}\right|^{2} d x^{\cdot}\right]^{1 / 2} \\
& \leqq\left[\int_{G / Z}\left(\int_{G / Z}\left|f\left(x y x^{-1}\right)\right| d y^{\cdot}\right)^{2} d x^{\cdot}\right]^{1 / 2} \cdot
\end{aligned}
$$

By Minkowski's integral inequality [2, p. 530] we can estimate the latter term by $\int_{G / Z}\left[\int_{G / Z}\left|f\left(y x y^{-1}\right)\right|^{2} d x^{*}\right]^{1 / 2} d y^{\circ}$. From unimodularity it follows that the inner integral equals $\|f\|_{x}^{2}$, so that $\left\|f^{\#}\right\|_{x} \leqq\|f\|_{x}$. Thus \# is a bounded linear operator $\mathscr{F}(\chi)_{2} \rightarrow \mathscr{F}(\chi)_{2}$, of norm $\leqq 1$, whose range is evidently $\mathscr{F}(\chi)_{2} \cap \mathscr{F}_{z}$. In fact, if $f \in \mathscr{F}(\chi)_{2} \cap \mathscr{F}_{z}$ then $f^{\#}=f$ a.e.

By Corollary (1.3), $\mathscr{F}_{c} \cap \mathscr{F}(\chi) \subseteq \mathscr{F}_{u}$. Moreover, Theorem (1.1) of [9] shows that

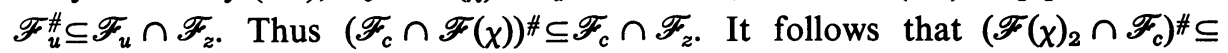
$\mathscr{F}(\chi)_{2} \cap \mathscr{F}_{z} \cap \mathscr{F}_{c}$.

CoRollary (4.3). $\left\{\chi_{\rho} \mid \rho \in \lambda^{-1}(x)\right\}$ is an orthonormal basis of $\mathscr{F}(\chi)_{2} \cap \mathscr{F}_{z}(G)$.

Proof. The results of $\S 1$ show that these functions are in $\mathscr{F}(\chi)_{2} \cap \mathscr{F}_{z}$ and are orthonormal in $\mathscr{F}(\chi)_{2}$. Let $f \in \mathscr{F}(\chi)_{2} \cap \mathscr{F}_{z}$ and choose $g \in \mathscr{F}(\chi)_{2} \cap \mathscr{F}_{c}$ so that $\|f-g\|_{x}<\varepsilon$. Then $(f-g)^{\#}=f^{\#}-g^{\#}=f-g^{\#}$. By the remarks above, $g^{\#} \in \mathscr{F}(\chi) \cap \mathscr{F}_{c}$ $\cap \mathscr{F}_{z}$. On the other hand, $\left\|f-g^{\#}\right\|_{x} \leqq\|f-g\|_{x}<\varepsilon$. Using Corollary (1.4), choose $\varphi=\sum_{k} c_{k} \chi_{\rho}{ }^{k}, \rho^{k} \in \mathscr{R}, c_{k} \in C$, so that $\left\|\varphi-g^{\#}\right\|_{G}<\varepsilon$. Then $\left\|\varphi-g^{\#}\right\|_{\chi} \leqq\left\|\varphi-g^{\#}\right\|_{G}<\varepsilon$ and therefore $\|\varphi-f\|_{\chi}<2 \varepsilon$.

Proof of the Plancherel Theorem. Let $f, g \in L_{1} \cap L_{2}(G)$. Then $f g^{-} \in L_{1}$, and therefore, by [16, p. 131], we have $\langle f, g\rangle=\int_{G / Z} \int_{z} f(x z) g(x z)^{-} d z d x, f_{x}$ and $g_{x} \in L_{1} \cap L_{2}(Z)$, and the inner integral exists for almost all $x$. By the Plancherel Theorem for abelian groups we have

$$
\int_{z} f_{x}(z) g_{x}(z)^{-} d z=\int_{z^{\wedge}}\left(f_{x}\right)^{\wedge}(\chi)\left(g_{x}\right)(\chi)^{-} d \chi
$$


Since

$$
\langle f, g\rangle=\int_{G / Z} \int_{Z^{\wedge}} P_{x}(f)(x) P_{x}(g)(x)^{-} d \chi d x
$$

is convergent and $(\chi, x) \rightarrow P_{\chi}(f)(x) P_{\chi}(g)(x)^{-}$is a function on $Z^{\wedge} \times G / Z$ we have, by the Fubini Theorem,

$$
\langle f, g\rangle=\int_{z^{\wedge}}\left\langle P_{\chi}(f), P_{\chi}(g)\right\rangle_{x} d x .
$$

We note that $P_{\chi}(f)$ (and $P_{\chi}(g)$ ) are in $\mathscr{F}(\chi)_{2}$. This follows from (iii) together with the Fubini Theorem if one takes $f=g$.

Since $\left\{d_{\rho}^{1 / 2} \rho_{i j} \mid \rho \in \lambda^{-1}(\chi)\right\}$ is an orthonormal basis of $\mathscr{F}(\chi)_{2}$, Parseval's equation tells us that

$$
\left\langle P_{\chi}(f), P_{\chi}(g)\right\rangle=\sum_{\rho \in \lambda-1(x): i, j=1, \ldots, d_{\rho}}\left\langle P_{\chi}(f), d_{\rho}^{1 / 2} \rho_{i j}\right\rangle_{\chi}\left\langle P_{\chi}(g), d_{\rho}^{1 / 2} \rho_{i j}\right\rangle_{\bar{x}}^{-} .
$$

Now

$$
\begin{aligned}
\left\langle P_{x}(f), d_{\rho}^{1 / 2} \rho_{i j}\right\rangle_{x} & =\int_{G / Z} P_{x}(f)(x) d_{\rho}^{1 / 2} \rho_{i j}(x)^{-} d x^{\cdot} \\
& =d_{\rho}^{1 / 2} \int_{G / Z}\left(\int_{Z} f(x z) \chi(z)^{-} d z\right) \rho_{i j}(x)^{-} d x
\end{aligned}
$$

which equals $d_{\rho}^{1 / 2} \int_{G / Z} \int_{z} f(x z) \rho_{i j}(x z)^{-} d z d x^{\circ}$, because $\rho \in \lambda^{-1}(\chi)$. Since the latter equals $d_{\rho}^{1 / 2} \int_{G} f(x) \rho_{i j}(x)^{-} d x$, it follows that

$$
\sum_{i, j=1}^{d_{\rho}}\left\langle P_{\chi}(f), d_{\rho}^{1 / 2} \rho_{i j}\right\rangle_{\chi}\left\langle P_{\chi}(g), d_{\rho}^{1 / 2} \rho_{i j}\right\rangle_{\bar{x}}^{-}=d_{\rho} \sum_{i, j=1}^{d_{\rho}} T_{f}\left(\rho^{-}\right)_{i j} T_{g}\left(\rho^{-}\right)_{i j} .
$$

This equals $d_{\rho} \operatorname{tr}\left(T_{f}\left(\rho^{-}\right) T_{g}\left(\rho^{-}\right)^{*}\right)$, where $\rho^{-}$is the irreducible unitary representation conjugate to $\rho$. Hence

$$
\left\langle P_{x}(f), P_{x}(g)\right\rangle_{x}=\sum_{\rho \in \lambda^{-1}(x)} d_{\rho^{-}} \operatorname{tr}\left(T_{f}\left(\rho^{-}\right) T_{g}\left(\rho^{-}\right)^{*}\right) .
$$

Equations (iv) and (v) give the Plancherel Formula with $\rho^{-}$in the place of $\rho$. However, since $\left(\rho^{-}\right)^{-}=\rho, \lambda\left(\rho^{-}\right)=\lambda(\rho)^{-}=\lambda(\rho)^{-1}$, the unimodularity of $Z$ implies that the Plancherel Formula as stated in Theorem (4.1) holds.

By using the Fourier transform $f^{\wedge}(\rho)$, we get a specialization of our result for central functions, as follows: As in $\S 3$ we see that, for $f$ in $\mathscr{Z}\left(L_{1}\right)$ and $\rho$ in $\mathscr{R}$, $T_{f}(\rho)=\lambda(\rho, f) I_{d_{\rho}}$. Thus, if $f, g \in \mathscr{Z}\left(L_{1}\right)$, then $T_{f}(\rho) T_{g}(\rho)^{*}=\lambda(\rho, f) \lambda(\rho, g)^{-} I_{d_{\rho}}$, so that $d_{\rho} \operatorname{tr}\left(T_{f}(\rho) T_{g}(\rho)^{*}\right)=d_{\rho}^{2} \lambda(\rho, f) \lambda(\rho, g)^{-}$. But $f^{\wedge}(\rho)=\lambda(\rho, f) d_{\rho}$; and since a similar equation holds for $g$ we see that, for each $\rho$,

$$
d_{\rho} \operatorname{tr}\left(T_{f}(\rho) T_{g}(\rho)^{*}\right)=f^{\wedge}(\rho) g^{\wedge}(\rho)^{-} .
$$

This gives 
Corollary (4.4) (The Plancherel Theorem for Central functions). Let $f$ and $g$ belong to $\mathscr{Z}\left(L_{1}\right) \cap L_{2}$. Then

$$
\langle f, g\rangle=\int_{Z^{\wedge}}\left(\sum_{\rho \in \lambda^{-1}(x)} f^{\wedge}(\rho) g^{\wedge}(\rho)^{-}\right) d x .
$$

The Plancherel Theorem (and its corollary for central functions) generalize and unify in a natural way the compact and abelian theorems: If $G$ is abelian then $d_{\rho}=1$ and $f^{\wedge}(\rho)=T_{f}(\rho)$, for all $\rho$. Moreover, $Z=G$ and $\mathscr{R}(G)=Z^{\wedge}$ so that $\lambda$ is the identity map. Thus $\lambda^{-1}(\chi)=\{\chi\}$, for $\chi \in Z^{\wedge}$. Writing $\chi$ instead of $\rho$, in (4.4), we have $\sum_{\rho \in \lambda^{-1}(x)} f^{\wedge}(\chi) g^{\wedge}(\chi)^{-}$which simply reduces to $f^{\wedge}(\chi) g^{\wedge}(\chi)^{-}$. Thus if

$$
f, g \in \mathscr{Z}\left(L_{1}\right) \cap L_{2}
$$

i.e., $L_{1} \cap L_{2}$, then $\langle f, g\rangle=\int_{G^{\wedge}} f^{\wedge}(\chi) g^{\wedge}(\chi)^{-} d \chi$. If $G$ is compact this is equivalent to $Z$ being compact or, alternatively, $Z^{\wedge}$ discrete. Thus Haar measure on $Z^{\wedge}$ is counting measure and the Plancherel Formula becomes

$$
\langle f, g\rangle=\sum_{x \in \mathbb{Z}_{\wedge}} \sum_{\rho \in \lambda^{-1}(x)} d_{\rho} \operatorname{tr}\left(T_{f}(\rho) T_{g}(\rho)^{*}\right) .
$$

This absolutely convergent sum can be rearranged in an arbitrary way. Since $\mathscr{R}(G)$ is partitioned into the disjoint union $\left\{\lambda^{-1}(x)\right\}_{x \in Z^{\wedge} \text {, we get }}$

$$
\langle f, g\rangle=\sum_{\rho \in \mathscr{R}_{(G)}} d_{\rho} \operatorname{tr}\left(T_{f}(\rho) T_{g}(\rho)^{*}\right) .
$$

These are the Plancherel Formulas of abelian and compact group theory, respectively.

The explicit formula for the Plancherel measure enables us to prove the following result.

COROLlary (4.5)(5). Let $G$ be a $[Z]$-group, $\mu_{\mathrm{pl}}$ the Plancherel measure and $\chi_{0} a$ fixed character in $Z^{\wedge}$. If $\lambda^{-1}\left(\chi_{0}\right)$ is open in $\mathscr{R}(G)$ or, more generally, if $\lambda^{-1}\left(\chi_{0}\right)$ has positive Plancherel measure, then $G$ is compact (and conversely). In particular, if $\mu_{\mathrm{pl}}\left(\rho_{0}\right)>0$, for any $\rho_{0} \in \mathscr{R}(G)$, then $G$ is compact.

Proof. If $\lambda^{-1}\left(\chi_{0}\right)$ is open then $\mu_{\mathrm{p} 1}\left(\lambda^{-1}\left(\chi_{0}\right)\right)>0$, by regularity. However,

$$
\mu_{\mathrm{pl}}\left(\lambda^{-1}\left(\chi_{0}\right)\right)=\operatorname{card}\left(\lambda^{-1}\left(\chi_{0}\right)\right) \int_{z^{\wedge}} \varphi_{\left\{x_{0}\right)}(x) d \chi .
$$

Unless $\mu_{Z^{\wedge}}\left(\chi_{0}\right)>0, \mu_{\mathrm{p} 1}\left(\lambda^{-1}\left(\chi_{0}\right)\right)=0$, a contradiction. Therefore, $\mu_{Z^{\wedge}}\left(\chi_{0}\right)>0$, and $Z^{\wedge}$ is discrete. Thus $Z$, and therefore $G$, are both compact.

For a [Z]-group $G$, we have shown in [9] that the function $w \in \mathscr{F}_{c_{0}}^{+}(G)$ has the following properties:

${ }^{(5)}$ The topology on $\mathscr{R}(G)$ as well as the regularity of the Plancherel measure will be discussed in $\$ 5$ and especially in $\$ 6$. 
(vi) $\int_{G} w(x) \rho_{i j}(x) \rho_{k l}(x)^{-} d x=d_{\rho}^{-1} \delta_{i k} \delta_{j l} \quad(\rho \in \mathscr{R}(G))$,

(vii) $\int_{G} w(x) \rho_{i j}(x) \sigma_{k l}(x)^{-} d x=0 \quad(\rho, \sigma \in \mathscr{R}(G), \rho \neq \sigma$, but $\lambda(\rho)=\lambda(\sigma))$.

It might be thought that, in addition, $w$ satisfies

$$
\int_{G} w(x) \rho_{i j}(x) \sigma_{k l}(x)^{-} d x=0, \text { for all } \rho \text { and } \sigma \in \mathscr{R}(G),
$$

where $\rho \neq \sigma$ (as is true in the compact case). The following result shows that this never happens unless $G$ is compact.

COROllary (4.6). Let $G$ be $a[Z]$-group and $w$ be an element of $\mathscr{F}_{c_{0}}(G)$. If $w$ satisfies (vi) and (viii) then $G$ is compact and $w \equiv 1$. Conversely, it is a classical fact (and our results imply) that, in the case of compact $G$, (vi) and (viii) hold for $w \equiv 1$, and only for this function.

Proof. Let $\rho \in \mathscr{R}$ and $\rho_{i j}$ be the coordinate functions with respect to some orthonormal basis. Then $\left(w \rho_{i j}\right)^{-} \in \mathscr{F}_{c_{0}}$. If $\sigma \in \mathscr{R}$ and $\sigma \neq \rho$ then

$$
T_{\left(w \rho_{i j}\right)}-(\sigma)=\int_{G}\left(w \rho_{i j}\right)^{-}(x) \sigma(x) d x=0, \quad \text { by (viii). }
$$

On the other hand,

$$
\left(T_{\left(w \rho_{i j}\right)}-(\rho)\right)_{k l}=d_{\rho} \delta_{i k} \delta_{j l} \quad \text { by (vi) }
$$

and therefore

$$
\left(T_{\left(w \rho_{i j}\right)}-(\rho) T_{\left(w \rho_{i j}\right)}-(\rho)^{*}\right)_{k l^{\prime}}=\sum_{l=1}^{d_{\rho}} d_{\rho}^{-2} \delta_{i k} \delta_{j l}^{2} \delta_{i l^{\prime}}=d_{\rho}^{-2} \delta_{i k} \delta_{i l^{\prime}}
$$

so that

$$
\operatorname{tr}\left(T_{\left(w \rho_{i j}\right)}-(\rho) T_{\left(w \rho_{i j}\right)}-(\rho)^{*}\right)=\sum_{k} d_{\rho}^{-2} \delta_{i k}^{2}=d_{\rho}^{-2}
$$

Also

$$
\operatorname{tr}\left(T_{\left(w \rho_{i j}\right)}-(\sigma) T_{\left(w \rho_{i j}\right)}-(\sigma)^{*}\right)=0, \text { for } \sigma \neq \rho .
$$

By the Plancherel Theorem we have

$$
\left\|w \rho_{i j}\right\|_{2}^{2}=\left\|\left(w \rho_{i j}\right)-\right\|_{2}^{2}=\int_{\mathscr{R}} d_{\sigma} \operatorname{tr}\left(T_{\left(w \rho_{i j}\right)}-(\sigma) T_{\left(w \rho_{i j}\right)}-(\sigma)^{*}\right) d \mu_{\mathrm{p} 1}(\sigma)=d_{\rho} d_{\rho}^{-2} \mu_{\mathrm{p} 1}(\rho) .
$$

Thus $\mu_{\mathrm{pl}}(\rho)=d_{\rho}\left\|w \rho_{i j}\right\|_{2}^{2}$. In particular, for $\rho=1$, we have $\mu(1)=\|w\|_{2}^{2}>0$ unless $w$ is trivial. On the other hand, (vi) shows that $w$ is nontrivial. We conclude from Corollary (4.5) that $G$ is compact, and thus (for the normalized Haar integral) we have $\int_{G} \rho_{i j}(x) \sigma_{k l}(x)^{-} d x=0$, for $\rho \neq \sigma$. Therefore $\int_{G}(w(x)-1) \rho_{i j}(x) \sigma_{k l}(x)^{-} d x=0$, for $\rho \neq \sigma$, by (viii). Letting $\sigma=1$, we conclude $T_{w-1}(\rho)=0$, for all $\rho \neq 1$. Also, $T_{w-1}(1)$ $=\int(w-1) d x=\int_{G} w d x-\mu(G)$. Since $\mu$ is normalized, and $\int w d x=1$ follows from 
(vi), we conclude $T_{w-1}(1)=0$. Therefore, by the Plancherel Theorem, $\|w-1\|_{2}^{2}=0$. Since $w$ and 1 are continuous, $w=1$.

We conclude the section with the remark that the Plancherel Formula and Corollary (4.4) give alternative proofs of the results of $\S 3$ in the case of [ $Z$ ]-groups.

5. Equivalence criteria for finite-dimensional unitary representations. We now study the possibility of generalizing Theorem (6.2), and its corollary, of [9], from $[Z]$-groups to arbitrary locally compact groups (in fact arbitrary topological groups) as well as the possibility of strengthening this result. It has been pointed out to us orally by J. Alperin and J. Thompson that this can be done for finite groups. Their result is contained in the following theorem valid for arbitrary topological groups. Moreover the constant $\sqrt{ } 2$ of the result in [9] referred to above can be replaced by $\sqrt{ } 3$ which, as was remarked in [9], is in any case best possible. In addition the conclusion holds for arbitrary finite-dimensional continuous unitary representations, not just irreducible ones.

THEOREM (5.1). Let $G$ be any topological group, $\rho$ and $\sigma$ finite-dimensional continuous unitary representations of $G$ satisfying $d_{\rho}=d_{\sigma}$ and $\left\|\chi_{\rho}-\chi_{\sigma}\right\|_{G}<\sqrt{ } 3$. Then $\rho$ and $\sigma$ are unitarily equivalent.

Proof. First assume $G$ is compact. As is well known, the conclusion holds iff $\chi_{\rho}=\chi_{\sigma}$. We may therefore assume $G$ is abelian; for if $x \in G$ and $H=[x]$ then $H$ is compact abelian, $\rho_{H}$ and $\sigma_{H}$ are as above i.e., $d_{\rho_{H}}=d_{\sigma_{H}}$ and $\left\|\chi_{\rho_{H}}-\chi_{\sigma_{H}}\right\|_{H}<\sqrt{ } 3$. We conclude from the abelian case that $\chi_{\rho_{H}}=\chi_{\sigma_{H}}$. In particular $\chi_{\rho}(x)=\chi_{\sigma}(x)$. Since $x$ is arbitrary, $\chi_{\rho}=\chi_{\sigma}$, and the result would follow. Hence, let $G$ be compact abelian and decompose $\rho$ and $\sigma$ into irreducible linear characters. By allowing the multiplicity to be 0 we may assume the same set of characters occur in each; i.e., $\rho=\sum_{i=1}^{r} \oplus n_{i} \chi_{i}$ and $\sigma=\sum_{i=1}^{r} \oplus m_{i} \chi_{i}$ where $\chi_{1}, \ldots, \chi_{r}$ are distinct elements of $G^{\wedge}$ and $m_{i}$ and $n_{i}$ are nonnegative integers. Then $\chi_{\rho}-\chi_{\sigma}=\sum_{i}\left(n_{i}-m_{i}\right) \chi_{i}$ so that $3>\left\|\chi_{\rho}-\chi_{\sigma}\right\|_{G}^{2} \geqq\left\|\chi_{\rho}-\chi_{\sigma}\right\|_{2}^{2}=\sum_{i}\left(n_{i}-m_{i}\right)^{2}$, by the orthogonality relations. Since $\sum_{i}\left(n_{i}-m_{i}\right)^{2}$ is an integer, it follows that it is $\leqq 2$; which, in turn, implies that $\left(n_{i}-m_{i}\right)^{2} \leqq 2$, for all $i$. That is $\left|n_{i}-m_{i}\right|$ is 0 or 1 . Since our task is to show $n_{i}=m_{i}$, for all $i$, we may ignore $i$ 's for which $\left|n_{i}-m_{i}\right|=0$. By the above, the number of $i$ 's for which $\left|n_{i}-m_{i}\right|=1$ is at most 2 . Thus we may assume $r \leqq 2$ and that $\left|n_{1}-m_{1}\right| \leqq 1$ and $\left|n_{2}-m_{2}\right| \leqq 1$. That is, $\chi_{\rho}=n_{1} \chi_{1}+n_{2} \chi_{2}, \chi_{\sigma}=m_{1} \chi_{1}+m_{2} \chi_{2}$. Since $\chi_{\rho}(1)=\chi_{\sigma}(1)$ we know $n_{1}+n_{2}=m_{1}+m_{2}$. If $\left|n_{1}-m_{1}\right|=0$ then $n_{1}=m_{1}$ and, by the above, $n_{2}=m_{2}$. Similarly, if $\left|n_{2}-m_{2}\right|=0$. Thus it is sufficient to assume $r=2$ and $\left|n_{1}-m_{1}\right|=1=$ $\left|n_{2}-m_{2}\right|$ and get a contradiction. Suppose $n_{1}>m_{1}$. Then $n_{1}-m_{1}=m_{2}-n_{2}=1$, and we have $\chi_{\rho}=\left(m_{1}+1\right) \chi_{1}+\left(m_{2}-1\right) \chi_{2}, \chi_{\sigma}=m_{1} \chi_{1}+m_{2} \chi_{2}$. Hence $\chi_{\rho}-\chi_{\sigma}=\chi_{1}-\chi_{2}$. That is, $\left\|\chi_{1}-\chi_{2}\right\|_{G}<\sqrt{ } 3$. Since $\chi_{1}$ and $\chi_{2}$ are distinct the proposition of $\S 6$ of [9] provides a contradiction.

This proves the theorem if $G$ is compact. Now let $G$ be any topological group and $\alpha: G \rightarrow G^{\alpha}$ be the canonical continuous homomorphism into its Bohr group. 
As is well known, $\rho$ and $\sigma$ induce finite-dimensional continuous unitary representations $\rho^{\alpha}$ and $\sigma^{\alpha}$ of $G^{\alpha}$ such that $\rho^{\alpha} \alpha=\rho$ and $\sigma^{\alpha} \alpha=\sigma$. Moreover, $\chi_{\rho^{\alpha}} \alpha=\chi_{\rho}$ and $\chi_{\sigma^{\alpha}} \alpha=\chi_{\sigma}$. Thus $d_{\rho^{\alpha}}=d_{\sigma^{\alpha}}$, and since $\alpha(G)$ is dense in $G^{\alpha},\left\|\chi_{\rho^{\alpha}}-\chi_{\sigma^{\alpha}}\right\|_{G^{\alpha}}<\sqrt{ } 3$. Hence $\rho^{\alpha}$ and $\sigma^{\alpha}$ are unitarily equivalent. It then follows that $\rho$ and $\sigma$ are also.

Corollary (5.2). Let $G$ be a [Z]-group and $\lambda: \mathscr{R}(G) \rightarrow Z^{\wedge}$ be the canonical map. Let $\mathscr{R}(G)$ be topologized by the compact-open topology on $\mathfrak{X}(G)$. Then, (1) for each $\chi \in Z^{\wedge}$, the set $\lambda^{-1}(\chi)$ is closed in $\mathscr{R}(G)$ and discrete in its relative topology, and (2) $\mathscr{R}(G)$ is a Hausdorff space.

Proof. Since $\lambda$ is evidently continuous and $Z^{\wedge}$ is a $T_{1}$-space, $\lambda^{-1}(\chi)$ is closed. Let $G=U Z$ where $U$ is a compact neighborhood, $\varepsilon>0$, and suppose $\rho_{v} \rightarrow \rho$ where $\rho_{\nu}$ and $\rho$ are in $\lambda^{-1}(\chi)$. For $\nu$ sufficiently large, we have $\left\|\chi_{\rho_{\nu}}-\chi_{\rho}\right\|_{U}<\varepsilon$. Let $x=u z$. Then $\chi_{\sigma}(u z)=\lambda_{\sigma}(u) \chi_{\sigma}(z)$, for any $\sigma$ in $\mathscr{R}$. Hence, since $\lambda(\rho)=\lambda\left(\rho_{v}\right)$ and $\left|\lambda_{\rho}(z)\right|=1$, we have $\left|\chi_{\rho}(x)-\chi_{\rho_{v}}(x)\right|=\left|\chi_{\rho}(u)-\chi_{\rho_{v}}(u)\right|$, so that $\left\|\chi_{\rho}-\chi_{\rho_{v}}\right\|_{G}=\left\|\chi_{\rho}-\chi_{\rho_{v}}\right\|_{U}<\varepsilon$. If $\varepsilon$ is sufficiently small then (1) follows from Theorem (5.1).

If $\rho_{1}$ and $\rho_{2}$ are distinct elements of $\mathscr{R}$ and if $\lambda\left(\rho_{1}\right) \neq \lambda\left(\rho_{2}\right)$ the facts that $\lambda$ is continuous and $Z^{\wedge}$ is a $T_{2}$-space imply that $\rho_{1}$ and $\rho_{2}$ can be separated by disjoint neighborhoods. Thus we may assume $\lambda\left(\rho_{1}\right)=\lambda\left(\rho_{2}\right)$. Let $\varepsilon$ and $U$ be as above and $W\left(U, \rho_{i}, \varepsilon\right)=\left\{\sigma \mid \sigma \in \mathscr{R}(G),\left\|\chi_{\sigma}-\chi_{\rho_{i}}\right\|_{U}<\varepsilon\right\}$ for $i=1,2$. Then each $W\left(U, \rho_{i}, \varepsilon\right)$ is a neighborhood of $\rho_{i}$. We show that they are disjoint, for $\varepsilon$ sufficiently small. If not, then $\left\|\chi_{\sigma}-\chi_{\rho_{i}}\right\|_{U}<\varepsilon$, for $i=1,2$ and some $\sigma \in \mathscr{R}(G)$. Therefore $\left\|\chi_{\rho_{1}}-\chi_{\rho_{2}}\right\|_{U} \leqq$ $\left\|\chi_{\rho_{1}}-\chi_{\sigma}\right\|_{U}+\left\|\chi_{\sigma}-\chi_{\rho_{2}}\right\|_{U}<2 \varepsilon$. Since $\lambda\left(\rho_{1}\right)=\lambda\left(\rho_{2}\right)$, we conclude from (1), by taking $\varepsilon$ sufficiently small, that $\rho_{1}=\rho_{2}$, a contradiction.

We shall see (5.2)(2) in another way in the next section where the topology on $\mathscr{R}(G)$ is studied in detail. Since we show in $\S 6$ that $\rho \rightarrow d_{\rho}$ is continuous it follows that the topology of uniform convergence on compacta of normalized characters is the same as that of the characters themselves. For if $\chi_{\rho_{v}} \rightarrow \chi_{\rho}$ uniformly on compacta then in particular $\chi_{\rho_{v}}(1) \rightarrow \chi_{\rho}(1)$. Therefore $d_{\rho_{v}} \rightarrow d_{\rho}$ and hence $\chi_{\rho_{v}} / d_{\rho_{v}}$ $\rightarrow \chi_{\rho} / d_{\rho}$ uniformly on compacta. On the other hand if $\chi_{\rho_{v}} / d_{\rho_{v}} \rightarrow \chi_{\rho} / d_{\rho}$ (c.o.) i.e. if $\rho_{\nu} \rightarrow \rho$ in $\mathscr{R}(G)$ then $d_{\rho_{v}} \rightarrow d_{\rho}$ by continuity. Therefore $\chi_{\rho_{v}} \rightarrow \chi_{\rho}$ (c.o.). Thus it follows that the $W(U, \rho, \varepsilon)$ in the above are neighborhoods of $\rho$ in $\mathscr{R}(G)$.

Next follows the formulation of some equivalent conditions.

Definition (5.3). Let $G$ be a locally compact group and $c>0$. We shall say $L_{1}(G)$ is abundant of type $c$ if for each pair of nonequivalent finite-dimensional unitary representations $\rho$ and $\sigma$ of $G$ there is an $f \in L_{1}(G)$, with $\|f\|_{1}=1$, such that

$$
\left|f^{\wedge}(\rho)-f^{\wedge}(\sigma)\right| \geqq c .
$$

We illustrate this notion with the following remark where we restrict the representations in the above definition to be irreducible and $G$ to be abelian. This method, which is a slight modification of that on p. 416 of [21], only works under the above conditions and only yields the constant $c=1$. Theorem (5.5) will both 
sharpen and generalize this result. Let $G$ be a locally compact abelian group, $\rho$ and $\sigma$ distinct characters. Then there exists an $f$ with $\|f\|_{1}=1$ such that

$$
\left|f^{\wedge}(\rho)-f^{\wedge}(\sigma)\right| \geqq 1 \text {. }
$$

To see this let $U$ be a compact symmetric neighborhood of 1 in $G$ such that $\rho U \cap \sigma U=\varnothing$. Then $0<\mu(U)<\infty$, where $\mu$ is Haar measure on $G^{\wedge}$. Since $\varphi_{U}$ and $\varphi_{\rho U^{-1}}$ are in $L_{2}\left(G^{\wedge}\right)$ there exist unique functions $g$ and $h$ in $L_{2}(G)$ so that $g^{\wedge}=\varphi_{U}$ and $h^{\wedge}=\varphi_{\rho U^{-1}}$, by the Plancherel Theorem. Let $f=g h$. Then $\|f\|_{1} \leqq\|g\|_{2}\|h\|_{2}$, $\|g\|_{2}=\left\|\varphi_{U}\right\|_{2}=\mu^{1 / 2}(U)$, and $\|h\|_{2}=\left\|\varphi_{\rho U^{-1}}\right\|_{2}=\mu^{1 / 2}\left(\rho U^{-1}\right)=\mu^{1 / 2}(U)$ since $\mu$ is Haar measure and $U$ is symmetric. Hence $\|f\|_{1} \leqq \mu(U)$. On the other hand, $f^{\wedge}=g^{\wedge} * h^{\wedge}$ $=\varphi_{U} * \varphi_{\rho U^{-1}}$. Since $U$ is symmetric we have, for $\gamma$ in $G^{\wedge}, \varphi_{U}\left(\gamma \chi^{-1}\right)=\varphi_{\gamma U^{-1}}(\chi)$, so $\left(\varphi_{U} * \varphi_{\rho U^{-1}}\right)(\chi)=\int_{G^{\wedge}} \varphi_{\gamma U^{-1}}(\chi) \varphi_{\rho U^{-1}}(\chi) d \mu(\chi)$. Thus $f^{\wedge}(\gamma)=\mu(\gamma U \cap \rho U)$. In particular, $f^{\wedge}(\rho)=\mu(U)>0$ and $f^{\wedge}(\sigma)=0$. Therefore $\left|f^{\wedge}(\rho)-f^{\wedge}(\sigma)\right|=\mu(U) \geqq\|f\|_{1}$. Since $f^{\wedge}(\rho)>0,\|f\|_{1}>0$, one obtains the result normalizing $f$.

Before stating the next theorem we require the following:

LEMMA (5.4). Let $\mu$ be a positive regular Borel measure and $f$ continuous bounded complex function on the locally compact space $X$. If $\|f\|_{X}>c>0$ then there is $a$ compact neighborhood $U$ in $X$ such that $\int_{U}|f|^{2} d \mu \geqq c \int_{U}|f| d \mu>0$.

Proof. Let $\varepsilon$ be any number satisfying $\frac{1}{2}\left(\|f\|_{X}-c\right)>\varepsilon>0$. Then $\|f\|_{X}^{2}-c\|f\|_{X}$ $\geqq 2 \varepsilon\|f\|_{X}$. Since $\left(\|f\|_{X}-\varepsilon\right)^{2} \geqq\|f\|_{X}^{2}-2 \varepsilon\|f\|_{X}$ we have

$$
c\|f\|_{X} \leqq\left(\|f\|_{X}-\varepsilon\right)^{2}
$$

also, evidently, $\|f\|_{X}>\varepsilon$. Choose an $x_{0} \in X$ so that $\|f\|_{X}-\varepsilon / 2 \leqq\left|f\left(x_{0}\right)\right| \leqq\|f\|_{X}$ and, by continuity, a compact neighborhood $U$ of $x_{0}$ so that ||$f(x)|-| f\left(x_{0}\right)|| \leqq$ $\left|f(x)-f\left(x_{0}\right)\right|<\varepsilon / 2$, for $x \in U$. Then we have $\|f\|_{X}-\varepsilon \leqq|f(x)| \leqq\|f\|_{X}$, in $U$. Since $c>0$ and $0<\mu(U)<\infty$, it follows that

$$
\mu(U)\left(\|f\|_{X}-\varepsilon\right)^{2} \leqq \int_{U}|f|^{2} d \mu \leqq \mu(U)\|f\|_{X}^{2}
$$

and

$$
c \mu(U)\left(\|f\|_{X}-\varepsilon\right) \leqq c \int_{U}|f| d \mu \leqq c \mu(U)\|f\|_{X}
$$

Since $c \mu(U)\left(\|f\|_{X}-\varepsilon\right)>0$, to complete the proof it suffices to show that

$$
\mu(U)\left(\|f\|_{X}-\varepsilon\right)^{2} \geqq c \mu(U)\|f\|_{X} .
$$

This is (i).

The next theorem generalizes and sharpens the discussion above dealing with the abelian case.

THEOREM (5.5). Let $G$ be a locally compact group and $0<c<\sqrt{ } 3$. Then $L_{1}(G)$ is abundant of type $c$. This result is best possible. 
Proof. Let $\rho$ and $\sigma$ be nonequivalent finite-dimensional continuous unitary representations. Then $\left\|\chi_{\rho}-\chi_{\sigma}\right\|_{G} \geqq \sqrt{ } 3$, by Theorem (5.1). So $0<c<\left\|\chi_{\rho}-\chi_{\sigma}\right\|_{G}$. By Lemma (5.4) there is a compact neighborhood $U$ in $G$ so that $\int_{U}\left|\chi_{\rho}-\chi_{\sigma}\right|^{2} d x$ $\geqq c \int_{U}\left|\chi_{\rho}-\chi_{\sigma}\right| d x$. Let $f=\varphi_{U}\left(\chi_{\rho}-\chi_{\sigma}\right)$. Then $f \in L_{1}$; in fact,

and

$$
\|f\|_{1}=\int_{U}\left|\chi_{\rho}-\chi_{\sigma}\right| d x \leqq\left\|\chi_{\rho}-\chi_{\sigma}\right\|_{G} \mu(U)<\infty
$$

$$
\begin{aligned}
\left|f^{\wedge}(\rho)-f^{\wedge}(\sigma)\right| & =\left|\int_{G} f(x)\left(\chi_{\rho}(x)-\chi_{\sigma}(x)\right)^{-} d x\right| \\
& =\int_{U}\left|\chi_{\rho}-\chi_{\sigma}\right|^{2} d x \geqq c \int_{U}\left|\chi_{\rho}-\chi_{\sigma}\right| d x=c\|f\|_{1} .
\end{aligned}
$$

Since the lemma tells us that $\|f\|_{1}>0$, it follows that $\left|g^{\wedge}(\rho)-g^{\wedge}(\sigma)\right| \geqq c$ where $g=f /\|f\|_{1}$ has $L_{1}$-norm 1 .

To see that the range $c<\sqrt{ } 3$ is the best possible we show that proving the result for any $c>0$ implies the conclusion of Theorem (5.1) for that $c$. The assertion of Theorem (5.5) then follows from the fact that $c<\sqrt{ } 3$ is best possible in the case of Theorem (5.1).

Indeed, suppose there is for each pair $\rho, \sigma$ an $L_{1}$-function $f$ with $\|f\|_{1}=1$ and $\left|f^{\wedge}(\rho)-f^{\wedge}(\sigma)\right| \geqq c$. Then $c \leqq\left|\int_{G} f(x)\left(\chi_{\rho}(x)-\chi_{\sigma}(x)\right)^{-} d x\right| \leqq\|f\|_{1}\left\|\chi_{\rho}-\chi_{\sigma}\right\|_{G}=$ $\left\|\chi_{\rho}-\chi_{\sigma}\right\|_{G}$. Thus the hypothesis $\left\|\chi_{\rho}-\chi_{\sigma}\right\|_{G}<c$ is false and the assertion of Theorem (5.1) is correct for that $c$.

In fact, we have proven that $L_{1}(G)$ is abundant of type $c$ if and only if the conclusion of Theorem (5.1) holds for that $c$.

COROLlaRY (5.6). Let $G$ be a connected locally compact abelian group and $0<c<2$. Then (1) if $\chi_{1}$ and $\chi_{2} \in G^{\wedge}$, and $\left\|\chi_{1}-\chi_{2}\right\|_{G}<c$ then $\chi_{1}=\chi_{2} ;(2)$ if $\chi_{1}$ and $\chi_{2}$ are distinct then there is a function $f$ with $\|f\|_{1}=1$ such that $\left|f^{\wedge}\left(\chi_{1}\right)-f^{\wedge}\left(\chi_{2}\right)\right| \geqq c$; (3) the range $c<2$ in (1) and (2) is best possible.

Proof. (1) was proven in $\S 6$ of [9] and $c<2$ was shown to be the best possible result. The remaining assertions follow from the remarks made above.

As is well known, the map $\rho \rightarrow T(\rho)$ which assigns to each continuous unitary representation (actually equivalence class) its Fourier transform is injective even if $\rho$ is infinite-dimensional. We observe that in the finite-dimensional case the same is true of the transform $f^{\wedge}(\rho)$ defined in $\S 3$.

COROLlaRY (5.7). Let $G$ be any locally compact group and $\rho$ and $\sigma$ continuous finite-dimensional unitary representations of $G$. If $f^{\wedge}(\rho)=f^{\wedge}(\sigma)$ for all $f$ in $L_{1}$, then $\rho$ and $\sigma$ are unitarily equivalent.

Definition (5.8). Let $c>0$ and $\left\{\lambda_{1}, \ldots, \lambda_{r}\right\}$ and $\left\{\mu_{1}, \ldots, \mu_{r}\right\}$ be 2-sets of (not necessarily distinct) numbers on the unit circle. Suppose the inequality

$$
\left|\sum_{i=1}^{r} \lambda_{i}^{n}-\mu_{i}^{n}\right|<c \text { for all } n \text { in } Z,
$$


implies that, after reordering of the indices, $\lambda_{i}=\mu_{i}$, for all $i$. Then we shall say we have a solution to the eigenvalue problem of type $c$.

THEOREM (5.9). If $0<c<\sqrt{ } 3$ then the eigenvalue problem has a solution. This range of values is best possible.

Proof. If $\lambda$ is on the unit circle then the map $n \rightarrow \lambda^{n}$ is a character of $Z$ which we denote by $\chi_{\lambda}$. Let $\rho=\sum_{i=1}^{r} \oplus \chi_{\lambda_{i}}$ and $\sigma=\sum_{i=1}^{r} \oplus \chi_{\mu_{i}}$. Then $\rho$ and $\sigma$ are continuous unitary representations of $Z$ of degree $r$ and, evidently, $\chi_{\rho}=\sum_{i=1}^{r} \chi_{\lambda_{i}}, \chi_{\sigma}=\sum_{i=1}^{r} \chi_{\mu_{i}}$. Thus

$$
\left\|\chi_{\rho}-\chi_{\sigma}\right\|_{Z}=\operatorname{lub}_{n}\left|\sum_{i=1}^{r} \lambda_{i}^{n}-\sum_{i=1}^{r} \mu_{i}^{n}\right| \leqq c<\sqrt{ } 3 .
$$

It follows from Theorem (5.1) that $\rho$ and $\sigma$ are unitarily equivalent. Since $\left\{\chi_{\lambda_{i}}\right\}_{i=1, \ldots, r}$ and $\left\{\chi_{\mu_{i}}\right\}_{i=1, \ldots, r}$ are the irreducible components and are 1-dimensional, after permutation of the $\mu_{i}$ we have $\chi_{\lambda_{i}}(n) \equiv \chi_{\mu_{i}}(n)$, for all $n$ and $i=1, \ldots, r$. One obtains the result by taking $n=1$.

We prove that this result is best possible by showing that, for any $c>0$, the conclusion of (5.9) is equivalent to that of Theorem (5.1). The result then follows from the fact that the conclusion of Theorem (5.1) is best possible.

Let $G$ be a topological group $\rho$ and $\sigma$ continuous finite-dimensional unitary representations of $G$ with $d_{\rho}=d_{\sigma}$. Let $c>0$ and $\left\|\chi_{\rho}-\chi_{\sigma}\right\|_{G}<c$. As in the proof of Theorem (5.1), if $x \in G$ and $H=\langle x\rangle$, then, evidently, $\rho_{H}$ and $\sigma_{H}$ are unitary representations of $H$, of equal degree, and $\left\|\chi_{\rho_{H}}-\chi_{\sigma_{H}}\right\|_{H}<c$. By semisimplicity $\rho_{H}=$ $\sum_{i=1}^{r} \oplus \chi_{i}, \sigma_{H}=\sum_{i=1}^{r} \oplus \xi_{i}$ where $\chi_{i}$ and $\xi_{i}$ are characters of $H$ and

$$
\left|\sum_{i=1}^{r} \chi_{i}(n x)-\sum_{i=1}^{r} \xi_{i}(n x)\right|<c
$$

for all $n$, so that $\left|\sum_{i=1}^{r}\left(\chi_{i}(x)\right)^{n}-\left(\xi_{i}(x)\right)^{n}\right|<c$, for all $n$. By the definition above, $\chi_{i}(x)=\xi_{i}(x)$, for all $i$, after suitable reordering. Hence $\chi_{\rho}(x)=\chi_{\sigma}(x)$. Since $x$ is arbitrary, $\chi_{\rho}=\chi_{\sigma}$, and therefore $\rho$ and $\sigma$ are unitarily equivalent.

Corollary (5.10). Let $A$ and $B$ be skew-Hermitian matrices of order $r$ and suppose

$$
\operatorname{lub}_{-\infty<t<\infty}|\operatorname{tr}(\exp t A)-\operatorname{tr}(\exp t B)|<\sqrt{ } 3
$$

Then $A$ and $B$ are unitarily similar.

Proof. Let $\rho(t)=\exp (t A), \sigma(t)=\exp (t B), t \in R$. Then $\rho$ and $\sigma$ are continuous unitary representations of $\boldsymbol{R}$ of degree $r$. The hypothesis tells us that $\left\|\chi_{\rho}-\chi_{\sigma}\right\|_{\boldsymbol{R}}$ $<\sqrt{ } 3$. Therefore $\rho$ and $\sigma$ are unitarily equivalent; thus there is a unitary operator $U$ satisfying $U \exp (t A) U^{-1}=\exp (t B)$, for all $t \in \boldsymbol{R}$. Hence, for all $t$,

\{eigenvalues of $\exp t A\}=\{$ eigenvalues of $\exp t B\}$.

If $\lambda_{1}, \ldots, \lambda_{r}$ and $\mu_{1}, \ldots, \mu_{r}$ denote the (nondistinct) eigenvalues of $A$ and $B$, 
respectively, it follows that $\{$ eigenvalues of $\exp t A\}=\left\{\exp \left[t \lambda_{1}\right], \ldots, \exp \left[t \lambda_{r}\right]\right\}=$ $\left\{\exp \left[t \mu_{1}\right], \ldots, \exp \left[t \mu_{r}\right]\right\}=\{$ eigenvalues of $\exp t B\}$, for all $t$. In particular,

$$
\left\{\exp \left[\lambda_{1}\right], \ldots, \exp \left[\lambda_{r}\right]\right\}=\left\{\exp \left[\mu_{1}\right], \ldots, \exp \left[\mu_{r}\right]\right\}
$$

Since exp is injective, $\left\{\lambda_{1}, \ldots, \lambda_{r}\right\}=\left\{\mu_{1}, \ldots, \mu_{r}\right\}$. On the other hand, since $A$ and $B$ are skew-Hermitian, they are diagonalizable by the spectral theorem.

REMARK. This result also follows from Theorem (5.9); we actually need only assume lub $-\infty<n<\infty|\operatorname{tr} \exp (n A)-\operatorname{tr} \exp (n B)|<\sqrt{ } 3$.

6. The structure of the algebras $L_{1}$ and $\mathscr{Z}\left(L_{1}\right)$.

THEOREM (6.1). Let $G$ be an [MAP]-group and $\mathscr{Z}\left(L_{1}\right)$ be the center of the group algebra. Then $\mathscr{Z}\left(L_{1}\right)$ is a commutative semisimple *algebra consisting of the central functions in $L_{1}(G)$.

$\operatorname{Proof}\left({ }^{6}\right)$. By [15], $G$ is unimodular. Hence the involution on $L_{1}$ is $f^{\vee}(x)=$ $f\left(x^{-1}\right)^{-}$. Also, $\mathscr{Z}\left(L_{1}\right)$ has the description referred to in $\$ 2$. Clearly, $\mathscr{Z}\left(L_{1}\right)$ is a closed subalgebra of $L_{1}$ which is $V$-stable and thus is a commutative *-algebra. If $\varphi=\chi_{\rho} / d_{\rho}$ where $\rho \in \mathscr{R}_{\mathrm{fn}}$, then $\varphi \in L_{\infty}$ and $L_{\varphi}$, the associated bounded linear function on $L_{1}$ gives, on restriction, a bounded linear functional on $\mathscr{Z}\left(L_{1}\right)$ of norm 1 . Moreover, since $\varphi=\varphi^{2}$ and $G$ is unimodular, we have

$$
\int_{G} f\left(x^{-1}\right)^{-} \varphi(x) d x=\int_{G} f(x)^{-} \varphi\left(x^{-1}\right) d x=\int_{G} f(x)^{-} \varphi(x)^{-} d x .
$$

Thus $L_{\varphi}\left(f^{\vee}\right)=L_{\varphi}(f)^{-}$and $L_{\varphi}$ is a *-functional. Finally, for any $f, g \in L_{1}$, we have $d_{\rho}^{-1} \operatorname{tr}\left(T_{f * g}(\rho)\right)=d_{\rho}^{-1} \operatorname{tr}\left(T_{f}(\rho) T_{g}(\rho)\right)$. Now if $f, g \in \mathscr{Z}\left(L_{1}\right)$, the proof of Corollary (3.3) shows that $T_{f}(\rho)=\lambda(f, \rho) I$ and $T_{g}(\rho)=\lambda(g, \rho) I$. Hence

$$
d_{\rho}^{-1} \operatorname{tr}\left(T_{f}(\rho) T_{\rho}(\rho)\right)=d_{\rho}^{-1} \operatorname{tr}\left(T_{f}(\rho)\right) d_{\rho}^{-1} \operatorname{tr}\left(T_{g}(\rho)\right),
$$

so that $L_{\varphi}(f * g)=L_{\varphi}(f) L_{\varphi}(g)$, for $f, g \in \mathscr{Z}\left(L_{1}\right)$, and $\left\{\chi_{\rho} / d_{\rho}: \rho \in \mathscr{R}_{\mathrm{fn}}\right\}$ give maximal ideals. Corollary (3.3) tells us that $\mathscr{Z}\left(L_{1}\right)$ is semisimple.

We now begin the study of the maximal ideal spaces $\mathscr{M}\left(L_{1}\right)$ and $\mathscr{M}\left(\mathscr{Z}\left(L_{1}\right)\right)$. Unless otherwise stated $G$ will always denote a $[Z]$-group.

Lemma (6.2). (1) If $f \in L_{1}, \varphi \in L_{\infty}$, then $\int_{G} f^{\sharp}(x) \varphi(x) d x=\int_{G} f(x) \varphi^{\sharp}(x) d x$. In particular, if $f \in \mathscr{Z}\left(L_{1}\right)$, then $L_{\varphi}(f)=L_{\varphi} \sharp(f)$, for each $\varphi$ in $L_{\infty}$.

(2) If $\varphi_{1}$ and $\varphi_{2} \in L_{\infty}$ and $L_{\varphi_{1}}=L_{\varphi_{2}}$ on $\mathscr{Z}\left(L_{1}\right)$ then $\varphi_{1}^{\#}=\varphi_{2}^{\#}$ locally a.e.

(3) If $\varphi_{1}$ and $\varphi_{2} \in \mathscr{F}_{c} \cap \mathscr{F}_{b} \cap \mathscr{F}_{z}$ and $L_{\varphi_{1}}=L_{\varphi_{2}}$ on $\mathscr{Z}\left(L_{1}\right)$ then $\varphi_{1}=\varphi_{2}$.

Proof. Taking into account the theorem on p. 131 of [16] we have

$$
\int_{G} f^{\#}(x) \varphi(x) d x=\int_{G}\left(\int_{G} w(t) f\left(t x t^{-1}\right) d t\right) \varphi(x) d x
$$

$\left({ }^{6}\right)$ For convenience, this result is stated for $[M A P]$-groups rather than [ $\left.Z\right]$-groups; however, in the case of $[Z]$-groups the proof of (6.1) is, of course, self-contained. Actually the statement of (6.1) is valid for arbitrary unimodular groups but we prefer the above formulation due to the explicit description which can be given of a separating family of maximal ideals. 
where $w$ is the weighting function. By the Fubini Theorem, the latter integral equals $\int_{G} w(t)\left(\int_{G} f\left(t x t^{-1}\right) \varphi(x) d x\right) d t$. By unimodularity this is

$$
\int_{G} w(t)\left(\int_{G} f(x) \varphi\left(t^{-1} x t\right) d x\right) d t .
$$

On reapplying the Fubini Theorem as well as the fact that $w$ can be chosen symmetric and that $G$ is unimodular one obtains $\int f(x) \varphi^{\#}(x) d x$.

(2) We may evidently assume $\varphi_{2}=0$. Thus $L_{\varphi_{1}}(f)=0$ for all $f \in \mathscr{Z}\left(L_{1}\right)$. By (1) this means that $L_{\varphi_{1}^{\#}}=0$ on $L_{1}$. Since $\varphi_{1}^{\#} \in L_{\infty}$ we have $\varphi_{1}^{\#}=0$ loc. a.e.

(3) Since $\varphi_{1}$ and $\varphi_{2} \in \mathscr{F}_{c} \cap \mathscr{F}_{z}, \varphi_{1}=\varphi_{1}^{\#}$ and $\varphi_{2}=\varphi_{2}^{\#}$. By (2), $\varphi_{1}^{\#}=\varphi_{2}^{\#}$ loc. a.e. Thus $\varphi_{1}=\varphi_{2}$ loc. a.e. Since these functions are continuous they are equal.

Lemma (6.3). (1) Let $\varphi \in \mathscr{F}_{u} \cap \mathscr{F}_{b}$ and $k(x, y)=\varphi(x y)-\varphi(x) \varphi(y)$ be defined on $G \times G$. Then $k \in \mathscr{F}_{u} \cap \mathscr{F}_{b}(G \times G)$.

(2) If $k$ is any function in $\mathscr{F}_{u} \cap \mathscr{F}_{b}(G \times G)$ and $f \in L_{1}(G)$ then the function $h$ defined by $h(y)=\int f(x) k(x, y) d x$ is in $\mathscr{F}_{c} \cap \mathscr{F}_{b}(G)$.

(3) Let $k$ be as in (2), $y$ be fixed in $G$ and $h_{1}(x)=\int_{G / Z} k\left(x, t y t^{-1}\right) d t^{\circ}$. Then $h_{1} \in$ $\mathscr{F}_{c} \cap \mathscr{F}_{b}(G)$.

(4) If $k$ is as in (2) and $\int_{G} \int_{G} f(x) g(y) k(x, y) d x d y=0$, for all $f, g \in \mathscr{Z}\left(L_{1}\right)$, then $\int_{G / Z} \int_{G / Z} k\left(s x s^{-1}, t y t^{-1}\right) d s^{*} d t^{\circ}=0$, for $x, y \in G$.

Proof. (1) It is evidently sufficient to show the functions $k_{1}(x, y)=\varphi(x y)$ and $k_{2}(x, y)=\varphi(x) \varphi(y)$ are in $\mathscr{F}_{u} \cap \mathscr{F}_{b}(G \times G)$. Evidently, $\left\|k_{1}\right\|_{G \times G} \leqq\|\varphi\|_{G},\left\|k_{2}\right\|_{G \times G}$ $\leqq\|\varphi\|_{G}^{2}$. The uniform continuity of $k_{2}$ follows easily from that of $\varphi$ together with the fact that $\|\varphi\|_{G}<\infty$, while the uniform continuity of $k_{1}$ follows from that of $\varphi$ together with the fact that $G \in[S I N]$.

(2) Clearly, $\|h\|_{G} \leqq\|f\|_{1}\|k\|_{G \times G}$. The continuity of $h$ follows readily from uniform continuity of $k$ and the fact that $\|f\|_{1}<\infty$.

(3) Since $\left\|h_{1}\right\|_{G} \leqq\|k\|_{G \times G}$, (3) follows from the uniform continuity of $k$.

(4) Let $h$ be as in (2). Then $\int_{G} g(y) h(y) d y=0$, for all $g \in \mathscr{Z}\left(L_{1}\right)$ and $h \in \mathscr{F}_{c} \cap \mathscr{F}_{b}$. It follows from Lemma (6.2) that $h^{\#}=0$, i.e. $\int_{G / Z} \int_{G} f(x) k\left(x, t y t^{-1}\right) d x d t^{\circ}=0$, for all $y$. This equals $\int_{G} f(x) \int_{G / Z} k\left(x, t y t^{-1}\right) d t^{*} d x$. Let $y$ be fixed and $h_{1}$ as in (3). Then $\int_{G} f(x) h_{1}(x) d x=0$, for all $f \in \mathscr{Z}\left(L_{1}\right)$ and $h_{1} \in \mathscr{F}_{c} \cap \mathscr{F}_{b}$. By Lemma (6.2), $h_{1}^{\#}=0$ in $x$. Thus $\int_{G / Z} \int_{G / Z} k\left(s x s^{-1}, t y t^{-1}\right) d s^{\cdot} d t^{\circ}=0$, for all $(x, y) \in G \times G$.

Theorem (6.4). Let $G$ be a $[Z]$-group. Then $\mathscr{M}\left(\mathscr{Z}\left(L_{1}\right)\right)$ coincides with $\mathfrak{X}(G)$, the set of normalized characters of $\rho$ 's in $\mathscr{R}(G)$.

Proof. The proof of Theorem (6.1) shows that, for each $\rho \in \mathscr{R}$, the function $\chi_{\rho} / d_{\rho}$ gives a distinct point in $\mathscr{M}\left(\mathscr{Z}\left(L_{1}\right)\right)$. For if

$$
\int_{G} f(x) d_{\rho}^{-1} \chi_{\rho}(x) d x=\int_{G} f(x) d_{\sigma}^{-1} \chi_{\sigma}(x) d x,
$$

for all $f \in \mathscr{Z}\left(L_{1}\right)$, then it follows from Lemma (6.2) that $\chi_{\rho} / d_{\rho}=\chi_{\sigma} / d_{\sigma}$, since these 
functions are continuous and central. The theorem of Frobenius and Schur then implies that $\rho=\sigma$. Evidently, if $\varphi=\chi_{\rho} / d_{\rho}$ then $\|\varphi\|_{G}=1=\left\|L_{\varphi}\right\|$.

Let $L$ be a nonzero bounded linear functional $\mathscr{Z}\left(L_{1}\right) \rightarrow C$ such that $L(f * g)$ $=L(f) L(g)$, for all $f, g \in \mathscr{Z}\left(L_{1}\right)$. Then $L$ extends, by the Hahn-Banach Theorem, to $\lambda \in L_{1}^{*}$ so that there is a $\varphi \in L_{\infty}$ satisfying $\lambda(f)=\int_{G} f(x) \varphi(x) d x$, for all $f \in L_{1}$. In particular, $L(f)=\int_{G} f(x) \varphi(x) d x$, for $f \in \mathscr{Z}\left(L_{1}\right)$, and we may assume $L=L_{\varphi}$. Choose $f_{0} \in \mathscr{Z}\left(L_{1}\right)$ for which $L_{\varphi}\left(f_{0}\right) \neq 0$. Then $L_{\varphi}(g)=L_{\varphi}\left(f_{0} * g\right) / L_{\varphi}\left(f_{0}\right)$, for all $g \in \mathscr{Z}\left(L_{1}\right)$. But

$$
L_{\varphi}\left(f_{0} * g\right)=\iint f_{0}(x) g\left(x^{-1} y\right) \varphi(y) d x d y=\iint f_{0}(x) g(y) \varphi(x y) d x d y .
$$

Thus if we let $\psi(y)=L_{\varphi}\left(f_{0}\right)^{-1} \int f_{0}(x) \varphi(x y) d x$, we have

$$
L_{\varphi}(g)=\int g(y) \psi(y) d y, \text { for all } g \in \mathscr{Z}\left(L_{1}\right)
$$

On the other hand, by unimodularity, $\psi=L_{\varphi}\left(f_{0}\right)^{-1}\left(f_{0}^{*}\right)^{v-*}$ ), so that $\psi \in \mathscr{F}_{u} \cap \mathscr{F}_{b}$ [16]. By (ii), $L=L_{\psi}$. By Lemma (6.2), $L_{\psi}=L_{\psi}$ and, by Theorem (2.1), $\left\|\psi^{\#}\right\|_{G} \leqq\|\psi\|_{G}$. Since $\psi^{\#}$ is in $\mathscr{F}_{u} \cap \mathscr{F}_{b} \cap \mathscr{F}_{z}$ we may assume $\varphi$ is also. Using (i) and the fact that $L_{\varphi}$ is multiplicative we have, by the Fubini Theorem,

$$
\iint f(x) f(y)(\varphi(x y)-\varphi(x) \varphi(y)) d x d y=0, \text { for all } f, g \in \mathscr{Z}\left(L_{1}\right) .
$$

Let $k(x, y)=\varphi(x y)-\varphi(x) \varphi(y)$. Then, by Lemma (6.3), it follows that $k \in \mathscr{F}_{u} \cap$ $\mathscr{F}_{b}(G \times G)$. By (4) of Lemma (6.3), we have

$$
\int_{G / Z} \int_{G / Z}\left(\varphi\left(s x s^{-1} t y t^{-1}\right)-\varphi\left(s x s^{-1}\right) \varphi\left(t y t^{-1}\right)\right) d s^{\cdot} d t^{\cdot}=0, \text { for all } x, y .
$$

Since $\varphi$ is central and $\mu_{G / Z}$ is normalized, this yields

$$
\int_{G / Z} \int_{G / Z} \varphi\left(s x s^{-1} t y t^{-1}\right) d s^{\cdot} d t^{\cdot}=\varphi(x) \varphi(y) .
$$

But the left side of (iii) equals $\int_{G / Z} \int_{G / Z} \varphi\left(x s^{-1} t y t^{-1} s\right) d t^{\circ} d s^{\circ}$, since $\varphi$ is central. That is, $\int_{G / Z} \int_{G / Z} \varphi\left(x s^{-1} t y\left(s^{-1} t\right)^{-1}\right) d t^{*} d s^{*}$, which equals $\int_{G / Z} \int_{G / Z} \varphi\left(x t y t^{-1}\right) d t^{*} d s^{*}$, by invariance. Since $\mu_{G / Z}$ is normalized we see that $\varphi$ satisfies the normalized character formula. The continuity and boundedness of $\varphi$ tells us, by Theorem (1.5), that $\varphi=\chi_{\rho} / d_{\rho}$, for a unique $\rho \in \mathscr{R}$.

Corollary (6.5). $\mathscr{Z}\left(L_{1}\right)$ is a commutative symmetric semisimple *-algebra, i.e., each $M$ in $\mathscr{M}\left(\mathscr{Z}\left(L_{1}\right)\right)$ is $\vee$-stable.

This follows from Theorem (6.1) and the fact that if $M=\operatorname{Ker} \varphi$ is a maximal ideal then $\varphi=\chi_{\rho} / d_{\rho}$, by Theorem (6.4), and in particular, $\varphi=\varphi^{\vee}$. The proof of Theorem (6.1) shows that $L_{\varphi}\left(f^{\vee}\right)=L_{\varphi}(f)^{-}$, for all $f \in \mathscr{Z}\left(L_{1}\right)$, so $M^{\vee}=M$. 
We denote by $\Phi(G) \subseteq L_{\infty}(G)$ the set of continuous normalized positive definite functions on the locally compact group $G$. As has been shown in Yoshizawa [28], the compact-open topology and the weak *-topology coincide on $\Phi$. A proof is also given in Dixmier [1] as well as in Godement [7] in the special case of [SIN]groups and the subset $\Phi_{0}-(0)$ of $\Phi$. Since $\mathfrak{X}$ is contained in $\Phi$, the same holds for $\mathfrak{X}$. It follows that the evaluation map $G \times \mathfrak{X} \rightarrow \boldsymbol{C}$ is jointly continuous.

We show that $\mathfrak{X} \cup(0)$ is $w^{*}$-closed in the strong unit sphere $S$ of $L_{\infty}$. It will then follow that $\mathfrak{X} \cup(0)$ is a compact $T_{2}$-space in the $w^{*}$-topology [16, p. 22] and, consequently, that $\mathfrak{X}$ is a locally compact $T_{2}$-space in the $w^{*}$-topology. It is not difficult to see that, in fact, $\mathfrak{X} \cup(0)$ is the one-point compactification of $\mathfrak{X}$. Suppose then that $\varphi_{\nu} \stackrel{w^{*}}{\rightarrow} \varphi, \varphi_{\nu} \in \mathfrak{X} \cup(0), \varphi \in S$. If (0) is confinal in $\varphi_{\nu}$, then evidently $\varphi=0$, and we are done. Thus we may assume $\varphi_{\nu} \neq 0$, for all $\nu$. Since $\varphi_{\nu}$ then is in $\Phi$ and since, as is well known [21], $\Phi$ is closed in $S$, we have $\varphi \in \Phi$. Thus $\varphi_{v} \rightarrow \varphi$ in the compact-open topology. Choose $\varepsilon>0$. Let $x, y \in G$ be fixed and consider the compact set $F x F^{-1} y$ where $F$ is any compact set containing $\{1\} \cup \operatorname{Supp} w$, and $w$ is the weighting function. Then $\varphi_{\nu}(x) \rightarrow \varphi(x), \varphi_{\nu}(y) \rightarrow \varphi(y)$,

$$
\begin{aligned}
\left|\int_{G / Z} \varphi_{\nu}\left(t x t^{-1} y\right) d t^{\cdot}-\int_{G / Z} \varphi\left(t x t^{-1} y\right) d t^{\cdot}\right| & \leqq \int_{G / Z}\left|\varphi_{\nu}\left(t x t^{-1} y\right)-\varphi\left(t x t^{-1} y\right)\right| d t^{\cdot} \\
& =\int_{G} w(t)\left|\varphi_{\nu}\left(t x t^{-1} y\right)-\varphi\left(t x t^{-1} y\right)\right| d t \leqq \varepsilon \int_{G} w(t) d t=\varepsilon .
\end{aligned}
$$

Thus $\int_{G / Z} \varphi_{\nu}\left(t x t^{-1} y\right) d t^{\circ} \rightarrow \int_{G / Z} \varphi\left(t x t^{-1} y\right) d t^{\circ}$. It follows that, since each $\varphi_{\nu}$ satisfies the normalized character formula so does $\varphi$; and since $\varphi$ is a continuous bounded function $(\varphi \in \Phi)$ we have, by Theorem (1.5), $\varphi \in \mathfrak{X}\left({ }^{7}\right)$.

Although the fact that $\rho \rightarrow d_{\rho}$ is continuous follows from the joint continuity of the evaluation map we prefer to give a direct proof as follows. If $\rho_{\nu} \rightarrow \rho$ in $\mathscr{R}(G)$ then $\chi_{\rho_{v}} / d_{\rho_{v}} \rightarrow \chi_{\rho} / d_{\rho}$ uniformly on compact sets of $G$. It follows that since $w$ is bounded

$$
w(x)\left|\chi_{\rho_{v}}(x)\right|^{2} d_{\rho_{v}}^{-2} \rightarrow w(x)\left|\chi_{\rho}(x)\right|^{2} d_{\rho}^{-2}
$$

uniformly on $U$, where $w$ is the weighting function and $G=U Z$ as above. Since supp $w \subseteq U$ it follows that

$$
\int_{G} w(x)\left|\chi_{\rho_{v}}(x)\right|^{2} d_{\rho_{\nu}}^{-2} d x \rightarrow \int_{G} w(x)\left|\chi_{\rho}(x)\right|^{2} d_{\rho}^{-2} d x
$$

By Corollary 2 of Theorem (2.2) of [9] we have $\int_{G} w(x)\left|\chi_{\sigma}(x)\right|^{2} d x=1$ for any $\sigma$ in $\mathscr{R}$. Thus $d_{\rho_{v}}^{-2} \rightarrow d_{\rho}^{-2}$ and therefore $d_{\rho_{v}} \rightarrow d_{\rho}$.

We summarize our results in

( ${ }^{7}$ ) An alternative proof is afforded by simply letting $f, g \in \mathscr{X}\left(L_{1}\right)$; then $f * g \in \mathscr{Z}\left(L_{1}\right)$. Since $\varphi_{\nu} \stackrel{w^{*}}{\rightarrow} \varphi$ we have $L_{\varphi_{v}} \rightarrow L_{\varphi}$ at $f, g$ and $f * g$. It follows that $L_{\dot{\omega}}$ is multiplicative on $\mathscr{Z}\left(L_{1}\right)$ and therefore, by Theorem (6.4), $\varphi \in \mathscr{X}$. ( $L_{\varphi}$ is nonzero because $\varphi \in \Phi$.) 
TheOREM (6.6). Let $G$ be $a[Z]$-group. Then on $\mathfrak{X}(G)$ the compact-open topology and $w^{*}$-topology coincide and the evaluation map $\mathfrak{X}(G) \times G \rightarrow C$ is jointly continuous. In this topology, $\mathfrak{X}(G)$ is a locally compact $T_{2}$-space whose one-point compactification is $\mathfrak{X}(G) \cup(0)$ (in the $w^{*}$-topology). Thus, if $\mathscr{M}\left(\mathscr{Z}\left(L_{1}\right)\right.$ ) is identified with $\mathfrak{X}(G)$, as in Theorem (6.4), the topology coincides with the Gelfand topology. Since $\mathfrak{X}(G)$ is in bijective correspondence with $\mathscr{R}(G)$, this gives a locally compact $T_{2}$-topology on $\mathscr{R}(G)$. The canonical map $\lambda: \mathscr{R} \rightarrow Z^{\wedge}$ is then evidently continuous and, as shown in $\S 5$, $\lambda^{-1}(\chi)$ is a closed discrete subset of $\mathscr{R}$ for each $\chi \in Z^{\wedge}$. Finally the map $\rho \rightarrow d_{\rho}$ is a continuous function on $\mathscr{R}$.

We now let $f \in L_{1}$ and modify the definition of Fourier transform by letting $f^{\sim}(\rho)=f^{\wedge}(\rho) / d \rho$ where $\rho \in \mathscr{R}(G)$. Then the continuity of the map $\rho \rightarrow f^{\sim}(\rho)$ of $\mathscr{R} \rightarrow C$ follows from the fact that the topology on $\mathscr{R}$ is the weak*-topology transferred from $\mathfrak{X}$. Since $\rho \rightarrow \infty$ in $\mathscr{R}$ means $\chi_{\rho} / d_{\rho} \stackrel{w^{*}}{\rightarrow} 0$ then evidently given $\varepsilon>0$ we have $\left|f^{\sim}(\rho)\right|<\varepsilon$ for $\rho$ in $\mathscr{R} \mid \mathscr{F}$ where $\mathscr{F}$ is a. suitable compact set. The inequality $\left|f^{\sim}(\rho)\right| \leqq\|f\|_{1}\left\|\chi_{\rho}\right\| d_{\rho}^{-1}=\|f\|_{1}$ shows that $\left\|f^{\sim}\right\|_{\mathscr{R}} \leqq\|f\|_{1}$. In addition one has

$$
\left(f^{\#}\right)^{\sim}(\rho)=\int_{G} f^{\#}(x) \chi_{\rho}(x) d_{\rho}^{-1} d x=\int_{G} \int_{G} w(t) f\left(t x t^{-1}\right) \chi_{\rho}(x) d_{\rho}^{-1} d x d t .
$$

By unimodularity of $G$, and the fact that $\chi_{\rho}$ is central, the latter equals

$$
\int_{G} \int_{G} w(t) f(x) d_{\rho}^{-1} \chi_{\rho}(x) d x d t=\int_{G} w(t) d t \int_{G} f(x) d_{\rho}^{-1} \chi_{\rho}(x) d x=f^{\sim}(\rho),
$$

so that $\sim$ may be thought of as defined only on $\mathscr{Z}\left(L_{1}\right)$; the same of course holds for $f^{\wedge}$. We now come to

Corollary (6.7) (The Riemann-Lebesgue Lemma). The map $\sim: L_{1}(G) \rightarrow \mathscr{F}_{c_{\infty}}(\mathfrak{X})$ is norm decreasing and the image $\mathfrak{A}$ of $L_{1}\left(\right.$ or $\left.\mathscr{Z}\left(L_{1}\right)\right)$ is a dense *-subalgebra of $\mathscr{F}_{c_{\infty}}(\mathfrak{X})$ (in the topology of uniform convergence).

Proof. The first assertion follows from the preceding remarks. In the course of the proof of (6.1) the equations $(f * g)^{\sim}(\rho)=f^{\sim}(\rho) g^{\sim}(\rho)$ and $\left(f^{\vee}\right)^{\sim}(\rho)=f^{\sim}(\rho)^{-}$ for $f, g \in \mathscr{Z}\left(L_{1}\right)$ were derived. Thus the map $f \rightarrow f^{\sim}(\rho)$ is a *-homomorphism of $\mathscr{Z}\left(L_{1}\right)$ for each $\rho$ in $\mathscr{R}$. It follows that $\mathfrak{A}$ is a ${ }^{*}$-subalgebra of $\mathscr{F}_{c_{\infty}}(\mathfrak{X})$. That $\mathfrak{A}$ vanishes at no point follows from Corollary (3.3). If $\rho$ and $\sigma$ are in $\mathscr{R}$ and $\rho \neq \sigma$ then $f^{\sim}(\rho) \neq f^{\sim}(\sigma)$ for some $f$ in $L_{1}$. If not then $\int f(x) \chi_{\rho}(x) d_{\rho}^{-1} d x=\int f(x) \chi_{\sigma}(x) d_{\sigma}^{-1} d x$ for all $f$ so that, as in the proof of (6.4), $\chi_{\rho} / d_{\rho}=\chi_{\sigma} / d_{\sigma}$. This dependence relation shows $\rho=\sigma$, a contradiction. The corollary now follows from the Stone-Weierstrass Theorem.

Corollary (6.8) (The UNIQUenesS OF THE INVERSE Fourier TRANSFORM). Let $\mu$ be a finite regular complex measure on $\mathfrak{X}(G)$. If $\int_{\mathfrak{X}(G)} d_{\rho}^{-1} \chi_{\rho}(x) d \mu(\rho)=0$, for all $x \in G$, then $\mu=0$. 
Proof. Let $f \in \mathscr{Z}\left(L_{1}\right)$. Then

$$
\begin{aligned}
\int_{\mathfrak{X}} f^{\sim}(\rho) d \mu(\rho) & =\int_{\mathfrak{X}} \int_{G} f(x) d_{\rho}^{-1} \chi_{\rho}(x) d x d \mu(\rho) \\
& =\int_{G} f(x) \int_{\mathfrak{X}} d_{\rho}^{-1} \chi_{\rho}(x) d \mu(\rho) d x=0 .
\end{aligned}
$$

Since $\mathfrak{A}$ is dense in $\mathscr{F}_{c_{\infty}}(\mathfrak{X})$, the result follows.

We note in passing where the "group characters" of $G$ fit into the picture.

Proposition (6.9). $\Phi_{0} \cap \mathscr{F}_{z}(G)=\left(G /\left(G^{\prime}\right)^{-}\right)^{\wedge}=\mathfrak{X}(G) \cap \Phi_{0}$, where $\Phi_{0}$ denotes the extreme points of $\Phi$.

Proof. The relations $\mathfrak{X} \cap \Phi_{0} \subseteq \mathscr{F}_{z} \cap \Phi_{0}$ and $\left(G /\left(G^{\prime}\right)^{-}\right)^{\wedge}=\left\{\rho \mid \rho \in \mathscr{R}, d_{\rho}=1\right\} \subseteq$ $\mathfrak{X} \cap \Phi_{0}$ are quite clear. It remains to prove $\Phi_{0} \cap \mathscr{F}_{z} \subseteq\left(G /\left(G^{\prime}\right)^{-}\right)^{\wedge}$. If $\varphi \in \Phi_{0}$ then $\varphi(x)=\left\langle\rho_{x}(v), v\right\rangle$ where $\rho \in \mathscr{R}_{\mathrm{fin}}$ and $\|v\|=1$ (see [7]). Extend $v$ to an orthonormal basis $\left\{v, v_{2}, \ldots, v_{n}\right\}$ of $V_{\rho}$. Since $\varphi \in \mathscr{F}_{z}$, we have

$$
\rho_{11}(x y)=\sum_{j=1}^{n} \rho_{1 j}(x) \rho_{j 1}(y)=\sum_{j=1}^{n} \rho_{1 j}(y) \rho_{j 1}(x)=\rho_{11}(y x) .
$$

It follows that $\sum_{j=2}^{n} \rho_{1 j}(x) \rho_{j 1}(y)-\sum_{j=2}^{n} \rho_{j 1}(x) \rho_{1 j}(y)=0$ for all $x, y$. By Burnside's Theorem (see Proposition (3.1) of [10]), we conclude $\rho_{j 1}(y)=\rho_{1 j}(y)=0$, for all $y \in G, j=2, \ldots, n$. Hence, unless $n=1, \rho$ is reducible; i.e., $\rho \in\left(G /\left(G^{\prime}\right)^{-}\right)^{\wedge}$.

As a consequence of our results we get a Bochner type theorem for central functions which generalizes the classical theorem for abelian groups [21, p. 410, Theorem 2].

THEOREM (6.10). Let $G$ be $a[Z]$-group and $\varphi$ be a continuous positive-definite central function. Then there exists a positive finite regular measure $d \mu_{\varphi}$ on $\mathfrak{X}(G)$ such that

$$
\varphi(x)=\int_{\mathfrak{X}(G)} \mathfrak{x}(x) d \mu_{\varphi}(\mathfrak{x}), \text { for all } x \in G .
$$

Conversely, each function defined by (iii), where $d \mu(\mathfrak{x})$ is any positive-finite regular measure on $\mathfrak{X}$, is continuous, positive-definite, and central. Thus (iii) sets up a bijective and, in fact, isometric correspondence between such functions and finite regular measures on $\mathfrak{X}(G)$.

Proof. Suppose $\varphi$ is defined by (iii) where $d \mu$ is a positive-finite regular measure. Then, since $\mathfrak{x}$ is in $\mathfrak{X}$, it is central and therefore, so is $\varphi$. Let $\lambda_{1}, \ldots, \lambda_{n}$ be complex numbers and $x_{1}, \ldots, x_{n} \in G$. Then $\sum_{i j} \lambda_{i}^{-} \lambda_{j} \varphi\left(x_{i}^{-1} x_{j}\right)=\int_{\mathfrak{X}} \sum_{i j} \lambda_{i}^{-} \lambda_{j} d_{\rho}^{-1} \chi_{\rho}\left(x_{i}^{-1} x_{j}\right) d \mu$. It follows from the fact that $\rho$ is a unitary representation that $\chi_{\rho} / d_{\rho}$ is positivedefinite; hence the integrand is nonnegative. Since $\mu$ is a positive measure $\varphi$ is positive-definite. Let $\varepsilon>0$ and choose a compact set $\mathscr{F} \subseteq \mathfrak{X}$ so that $\mu(\mathfrak{X} \mid \mathscr{F})<\varepsilon$. Since $\mathscr{F}$ is compact the Ascoli Theorem tells us $\mathscr{F}$ is equicontinuous. For $x_{0} \in G$, 
choose a neighborhood $U$ of $x_{0}$ so that if $x \in U$ then $\left|\mathfrak{x}(x)-\mathfrak{x}\left(x_{0}\right)\right|<\varepsilon$, for all $\mathfrak{x} \in \mathscr{F}$. For $x$ in $U$, we have

$$
\left|\varphi(x)-\varphi\left(x_{0}\right)\right| \leqq \int_{\mathfrak{X} \mid \mathscr{F}}\left|\mathfrak{x}(x)-\mathfrak{x}\left(x_{0}\right)\right| d \mu(\mathfrak{x})+\int_{\mathscr{F}}\left|\mathfrak{x}(x)-\mathfrak{x}\left(x_{0}\right)\right| d \mu(\mathfrak{x}) \leqq \varepsilon(\|\mu\|+2),
$$

because the r's are normalized. Thus $\varphi$ is continuous.

For the converse, $\mathscr{Z}\left(L_{1}\right)$ is a commutative semisimple *-algebra, and, as is well known, $L_{\varphi}$ is a positive bounded linear functional on $L_{1}$ which is extendable and respects involutions [21]. In particular, this is true on $\mathscr{Z}\left(L_{1}\right)$. We have, by the theorem of Herglotz-Bochner-Weil-Raikov [16, p. 97], that there exists a positive finite regular measure $\mu_{\varphi}$ on $\mathscr{M}\left(\mathscr{Z}\left(L_{1}\right)\right)$, i.e. $\mathfrak{X}$, such that $L_{\varphi}(f)=\int_{\mathfrak{X}} f^{\sim}(\mathfrak{x}) d \mu_{\varphi}(\mathfrak{X})$, where $f^{\sim}(\mathfrak{x})=L_{\mathfrak{x}}(f), \mathfrak{x} \in \mathfrak{X}$. Thus $\int_{G} f(x) \varphi(x) d x=\int_{\mathfrak{X}} \int_{G} f(x) \mathfrak{x}(x) d x d \mu_{\varphi}(\mathfrak{x})$. By the Fubini Theorem, $\int_{G} f(x) \varphi(x) d x=\int_{G} f(x) \psi(x) d x$, for all $f \in \mathscr{Z}\left(L_{1}\right)$, where $\psi(x)=$ $\int_{\mathfrak{X}} \mathfrak{x}(x) d \mu_{\varphi}(\mathfrak{x})$. Since, by the part of the theorem already proven, $\psi$ is continuous and central and since these are the assumptions on $\varphi$, it follows from Lemma (6.2) that $\varphi(x)=\int_{\mathfrak{X}} \mathfrak{x}(x) d \mu_{\varphi}(\mathfrak{x})$, for all $x \in G$. Finally, for $x=1$, we get $\varphi(1)=\int_{\mathfrak{X}} \mathfrak{x}(1) d \mu_{\varphi}(\mathfrak{x})$ $=\mu_{\varphi}(\mathfrak{X})$, since the $\mathfrak{x}$ 's are normalized. But $\varphi$ is positive-definite, so that $\|\varphi\|_{G}=\varphi(1)$ $=\left\|\mu_{\varphi}\right\|$.

The following inversion theorem follows from our Bochner Theorem and an abstract type inversion theorem [16, p. 99] in much the same way that the inversion theorem for abelian groups does [16, p. 143]. In this connection, see Lemma (2.3), Corollary (6.5) and Corollary (6.7). In what follows [ ] will denote complex linear span.

Actually, because of the form of Bochner's Theorem as well as the fact that the maps $f \rightarrow f^{\sim}(\rho)$ are the *-algebra homomorphisms of $\mathscr{Z}\left(L_{1}\right)$, the Inversion formula is given in the form: there is a unique positive regular measure $d v$ on $\mathfrak{X}$ such that for each $f$ in $\left[\mathscr{Z}\left(L_{1}\right) \cap \Phi\right]$ one has $f(x)=\int_{\mathfrak{X}} \chi_{\rho}(x)^{-} d_{\rho}^{-1} f^{\sim}(\rho) d \nu(\rho)$. However this equals $\int_{\mathfrak{X}} \chi_{\rho}(x)^{-} d_{\rho}^{-2} f^{\wedge}(\rho) d \nu(\rho)$. Since $\rho \rightarrow d_{\rho}^{-2}$ is a nonnegative continuous function on $\mathfrak{X}$ and $d \nu$ is a positive regular measure, the Riesz theorem shows the same is true of $d_{\rho}^{-2} d \nu(\rho)$. The modified $d \nu$ turns out to be $d \mu_{\mathrm{pl}}$ up to a positive constant. (As was remarked in $\S 4, \mu_{\mathrm{pl}}$ is determined, as in the abelian case, only up to a normalization.)

THEOREM (6.11) (Fourier INVERSION THEOREM FOR CENTRAL FUNCTIONS) $\left({ }^{8}\right)$. The Plancherel measure $d \mu_{\mathrm{pl}}$ is the unique regular measure on $\mathfrak{X}(G)$ such that for each $f$ in $\left[\mathscr{Z}\left(L_{1}\right) \cap \Phi\right]$ the function $\rho \rightarrow f^{\wedge}(\rho) d \rho$ is in $L_{1}\left(\mathfrak{X}, d \mu_{\mathrm{pl}}\right)$ and

$$
f(x)=\int_{\mathfrak{X}} f^{\wedge}(\rho) \chi_{\rho}(x)^{-} d \mu_{\mathrm{pl}}(\rho), \quad x \in G .
$$

In particular, this will establish the uniqueness of the Plancherel measure. In fact,

$\left.{ }^{8}\right)$ In a later publication we shall prove a general Fourier Inversion Theorem (which depends on (6.1)) for functions which are not necessarily central. 
the Plancherel measure is uniquely characterized by the property expressed in Corollary (4.4). As the remarks preceding the theorem indicate, there exists a unique regular measure $d \nu$ satisfying the condition of Theorem (6.11). It remains to show $d \nu=d \mu_{\mathrm{pl}}$.

Proof. If $g \in \mathscr{Z}\left(L_{1}\right) \cap L_{2}$ and $f=g * g^{\vee}$ then $f$ is continuous, positive-definite, and in $\mathscr{Z}\left(L_{1}\right)$. The Inversion Theorem applies to $f$. Thus $f(1)=\int_{\mathscr{R}} d_{\rho} f^{\wedge}(\rho) d v(\rho)$. On the other hand $f(1)=\|g\|_{2}^{2}$, and by the results of $\S 3 d_{\rho} f^{\wedge}(\rho)=\left|g^{\wedge}(\rho)\right|^{2}$, so that $\|g\|_{2}^{2}=\int\left|g^{\wedge}(\rho)\right|^{2} d \nu$. However, by the Plancherel Theorem for central functions, $\|g\|_{2}^{2}=\int\left|g^{\wedge}(\rho)\right|^{2} d \mu_{\mathrm{pl}}$. Hence, for all $g$ in $\mathscr{Z}\left(L_{1}\right) \cap L_{2}$ one has

$$
\int_{\mathscr{R}}\left|g^{\wedge}(\rho)\right|^{2} d \nu=\int_{\mathscr{R}}\left|g^{\wedge}(\rho)\right|^{2} d \mu_{\mathrm{pl}}<\infty
$$

Let $\mathfrak{A}_{1,2}=\left\{g^{\wedge} \mid g \in \mathscr{Z}\left(L_{1}\right) \cap L_{2}\right\}$ and $d \mu$ be either $d \mu_{\mathrm{pl}}$ or $d \nu$. We show the subspace $\mathfrak{A}_{1,2}$ is dense in $L_{2}(\mathfrak{X}, d \mu)$. To this end let $h$ be in $L_{2}(\mathfrak{X}, d \mu)$ and suppose $\int_{\mathfrak{X}} g^{\wedge}(\rho) h(\rho)^{-} d \mu=0$ for all $g^{\wedge}$ in $\mathfrak{A}_{1,2}$. Then since $\mathfrak{A}_{1,2}=\left\{g^{\wedge} \mid g \in L_{1} \cap L_{2}\right\}$ and, as was remarked in $\S 3,\left(g_{x^{-1}}\right)^{\wedge}(\rho)=d_{\rho}^{-1} \chi_{\rho}(x) g^{\wedge}(\rho)$ for all $g$ in $\mathscr{Z}\left(L_{1}\right)$ we have $\int \mathfrak{X} d_{\rho}^{-1} \chi_{\rho}(x) g^{\wedge}(\rho) h(\rho)^{-} d \mu$ for all $g$ in $\mathscr{Z}\left(L_{1}\right) \cap L_{2}$ and $x \in G$. But $g^{\wedge} \in L_{2}(\mathfrak{X}, d \mu)$ by the above, and $h \in L_{2}(\mathfrak{X}, d \mu)$ by assumption. Therefore $g^{\wedge} h^{-} \in L_{1}(\mathfrak{X}, d \mu)$ and by the uniqueness of the inverse Fourier transform, $g^{\wedge} h^{-}=0$ a.e. on $\mathfrak{X}$. For each $\rho \in \mathfrak{X}$ there exists a $g$ in $\mathscr{F}_{c_{0}} \cap \mathscr{F}_{z}$ so that $g^{\wedge}(\rho) \neq 0$ and by continuity (since $g^{\sim}$ and $\rho \rightarrow d_{\rho}$ are continuous so is $g^{\wedge}$ ) a neighborhood $\mathscr{U}_{\rho}$ of $\rho$ on which $g^{\wedge} \neq 0$. To see this first choose by the above a $g$ in $\mathscr{Z}\left(L_{1}\right)$ so that $g^{\wedge}(\rho) \neq 0$ and then a sequence $g_{n}$ in $\mathscr{F}_{c_{0}}$ such that $g_{n} \rightarrow g$ in $L_{1}$. Since \# is a bounded operator we have $g_{n}^{\#} \rightarrow g^{\#}$ in $L_{1}$. On the other hand $g_{n}^{\#} \in \mathscr{F}_{c_{0}} \cap \mathscr{F}_{z}$ and $g=g^{\#}$. Therefore we have a sequence, which we again call $g_{n}$, in $\mathscr{F}_{c_{0}} \cap \mathscr{F}_{z} \rightarrow g$ in $L_{1}$. It follows that $g_{n}^{\tilde{n}} \rightarrow g^{\sim}$ uniformly on $\mathfrak{X}$ and in particular, at $\rho$. Multiplying by $d_{\rho}$ we conclude that $\widehat{g_{n}}(\rho) \rightarrow g^{\wedge}(\rho)$. Since $g^{\wedge}(\rho) \neq 0$ it follows that there is some $n$ so that $g_{n}(\rho) \neq 0$. Consequently $h=0$ a.e. on each $\mathscr{U}_{\rho}$. On the other hand because $h \in L_{2}(\mathfrak{X}, d \mu)$ and $d \mu$ is regular, $h$ has (up to a null set) $\sigma$-compact support. The set $\left\{\mathscr{U}_{\rho} / \rho \in \mathscr{R}\right\}$ is an open covering of $\mathfrak{X}$ and therefore supp $h \subseteq \bigcup_{i=1}^{\infty} \mathscr{U}_{\rho_{i}}$ for some countable number of $\rho_{i}$ 's. That $h=0$ a.e. on $\mathfrak{X}$ now follows from the fact that $h=0$ a.e. on each $\mathscr{U}_{\rho_{i}}$.

The identity map from $\mathfrak{A}_{1,2}$ to itself regarded both as a subspace of $L_{2}\left(\mathfrak{X}, d \mu_{\mathrm{p} 1}\right)$ and $L_{2}(\mathfrak{X}, d v)$ extends to an isometry $I: L_{2}\left(\mathfrak{X}, d \mu_{\mathrm{pl}}\right) \rightarrow L_{2}(\mathfrak{X}, d v)$. From this it follows that a Borel set $\mathscr{S}$ of $\mathfrak{X}$ has finite $\mu_{\mathrm{pl}}$ measure iff it has finite $\nu$ measure and $\mu_{\mathrm{pl}}(\mathscr{S})=\nu(\mathscr{S})$; i.e., $\mu_{\mathrm{pl}}=\nu$.

Corollary (6.12). The Fourier transform $f \rightarrow f^{\wedge}$, where $f \in \mathscr{Z}\left(L_{1}\right) \cap L_{2}$, extends to an isometric linear map $L_{2}^{\#}(G) \rightarrow L_{2}\left(\mathfrak{X}, d \mu_{\mathrm{pl}}\right)$ where $L_{2}^{\#}$ is defined in $\S 2$.

Proof. Evidently, $L_{2}^{\#}$ is the closure of $\mathscr{Z}\left(L_{1}\right) \cap L_{2}$ in $L_{2}$. Since $\mathfrak{A}_{1,2}$ is dense in $L_{2}\left(\mathfrak{X}, d \mu_{\mathrm{pl}}\right)$ the isometric linear surjection $\mathscr{Z}\left(L_{1}\right) \cap L_{2} \rightarrow \mathfrak{A}_{1,2}$ extends in a unique way to an isometry of $L_{2}^{\#}(G)$ onto $L_{2}\left(\mathfrak{X}, d \mu_{\mathrm{pl}}\right)$. 
We now turn to the $L_{1}$-algebra and its maximal ideal space $\mathscr{M}\left(L_{1}\right)$ and study the relationship with $\mathscr{Z}\left(L_{1}\right)$ and $\mathscr{M}\left(\mathscr{Z}\left(L_{1}\right)\right)$, respectively. This carries out, for the analogous situation of a group algebra (of a [Z]-group), the program introduced by $\mathrm{Y}$. Misonou and M. Nakamura in [20] for $C^{*}$-algebras and uses, particularly in Lemma (6.15), a variant of Godement's technique [6, Lemma 15].

Proposition (6.13). $L_{1}(G)$ has an approximate identity consisting of central functions.

This result follows immediately from Theorem (1.3) of [9].

Lemma (6.14). If $I$ is a proper closed 2-sided ideal in $L_{1}$ then $I \cap \mathscr{Z}\left(L_{1}\right) \neq \mathscr{Z}\left(L_{1}\right)$.

Proof. Otherwise, $I \supseteq \mathscr{Z}\left(L_{1}\right)$. Let $f_{v}$ be an approximate identity in $L_{1}$ consisting of elements in $\mathscr{Z}\left(L_{1}\right)$. For $f \in L_{1}$, we have $f * f_{v} \rightarrow f$ in $L_{1}$. Hence $f \in I$, since $I$ is a closed ideal. Thus $I=L_{1}$.

LEMMA (6.15). Each $I \in \mathscr{M}\left(L_{1}\right)$ has the property that

$$
I=\left\{f \mid(f * g)^{\#} \in I \text { for all } g \in L_{1}\right\} .
$$

Proof. Let the latter set be denoted by $J_{I}$. If $f \in I$ and $g \in L_{1}$ then $f * g \in I$ and therefore $(f * g)^{\#} \in I^{\#} \subseteq I$, by Theorem (2.2). Thus $I \subseteq J_{I}$. Next we show $J_{I}$ is a 2-sided ideal. Clearly $J_{I}$ is a subspace of $L_{1}$. If $f \in J_{I}$ and $h \in L_{1}$ then $((f * h) * g)^{\#}$ $=(f *(h * g))^{\#} \in I$, so $f * h \in J_{I}$. Also $((h * f) * g)^{\#}$ equals, by Theorem (2.1), $(g *(h * f))^{\#}=((g * h) * f)^{\#}=(f *(g * h))^{\#}$, again by Theorem (2.1). The latter is in $I$, so $h * f$ is in $J_{I}$, and $J_{I}$ is a 2-sided ideal.

Since $I$ is maximal, $J_{I}=I$ or $J_{I}=L_{1}$. If $J_{I}=L_{1}$ then, for any $f$ and $g \in L_{1}$, we have $(f * g)^{\#}$ in $I$ and therefore in $I \cap \mathscr{Z}\left(L_{1}\right)$. Let $e$ be the identity modulo $I$. Then $(e * g)^{\#} \in I \cap \mathscr{Z}\left(L_{1}\right)$ and $e * g-g \in I$, for all $g \in L_{1}$. It follows that $(e * g)^{\#}-g^{\#} \in I^{\#}$ $=I \cap \mathscr{Z}\left(L_{1}\right)$ and therefore $g^{\#} \in I \cap \mathscr{Z}\left(L_{1}\right)$, for all $g \in L_{1}$, i.e., $\mathscr{Z}\left(L_{1}\right)=I \cap \mathscr{Z}\left(L_{1}\right)$. This contradicts Lemma (6.14).

We evidently get the following corollary.

Corollary (6.16). If $I$ and $I^{\prime}$ are in $\mathscr{M}\left(L_{1}\right)$ then $I=I^{\prime}$ iff $I \cap \mathscr{Z}\left(L_{1}\right)=I^{\prime} \cap \mathscr{Z}\left(L_{1}\right)$.

Proposition (6.17). If I is a regular maximal 2-sided ideal in $L_{1}$ then $I \cap \mathscr{Z}\left(L_{1}\right)$ is such an ideal in $\mathscr{Z}\left(L_{1}\right)$.

Proof. I $\mathscr{Z}\left(L_{1}\right)$ is clearly an ideal in $\mathscr{Z}\left(L_{1}\right)$. If $e$ is the identity modulo $I$ then $e^{\#} \in \mathscr{Z}\left(L_{1}\right)$ and $e * f^{\#}-f^{\#} \in I$, for all $f^{\#}$ in $\mathscr{Z}\left(L_{1}\right)$. It follows from Theorems (2.1) and (2.2) that $\left(e * f^{\#}-f^{\#}\right)^{\#}=\left(e * f^{\#}\right)^{\#}-f^{\# \#}=e^{\#} * f^{\#}-f^{\#} \in I^{\#}=I \cap \mathscr{Z}\left(L_{1}\right)$. Therefore $I^{\#}$ is regular. We show it is maximal. If not, then, by regularity, we can imbed it in a maximal regular ideal $M$ of $\mathscr{Z}\left(L_{1}\right)$. Then, as in Lemma (6.15), $J_{M}$ is a 2-sided ideal in $L_{1}$ containing $I$. In addition, $J_{M} \neq L_{1}$; otherwise, $(f * g)^{\#} \in M$, for all $f, g \in L_{1}$. In particular, $\left(e^{\#} * g\right)^{\#}=e^{\#} * g^{\#} \in M$, for all $g \in L_{1}$, and since $e^{\#} * g^{\#}-g^{\#} \in I \subseteq M$,

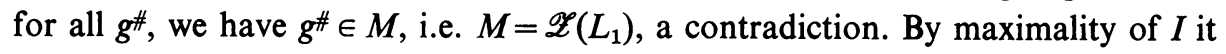


follows that $J_{M}=I$. Let $f^{\#} \in M$, where $f \in L_{1}$. Then $\left(f^{\#} * g\right)^{\#}=f^{\#} * g^{\#} \in M$, for all $g \in L_{1}$ since $M$ is an ideal. Therefore, $f^{\#} \in I \cap \mathscr{Z}\left(L_{1}\right)$. Thus $M \subseteq I^{\#}$. This means that $M=I^{\#}$ and therefore $I^{\#}$ is maximal.

The following theorem generalizes a result of I. E. Segal concerning compact groups [22]; for abelian groups it is well known.

TheOREM (6.18). Let $G$ be $a[Z]$-group and $I \in \mathscr{M}\left(L_{1}\right)$. Then $I=\operatorname{Ker} T(\rho)$, for $a$ unique $\rho \in \mathscr{R}(G)$. Conversely, each $\rho$ in $\mathscr{R}(G)$ gives a regular maximal 2-sided ideal in this way.

Proof. That $\operatorname{Ker} T(\rho)$ is a regular maximal 2-sided ideal was shown in the proof of Theorem (3.1). Let $I$ be such an ideal. Then $I \cap \mathscr{Z}\left(L_{1}\right)$ is in $\mathscr{M}\left(\mathscr{Z}\left(L_{1}\right)\right)$, by Proposition (6.17). By Theorem (6.4), there exists a $\rho \in \mathscr{R}$ so that $I \cap \mathscr{Z}\left(L_{1}\right)$ $=\left\{f \mid f \in \mathscr{Z}\left(L_{1}\right), f^{\wedge}(\rho)=0\right\}$. Consider $T(\rho)$, its Fourier transform. Then $I_{\rho}=\operatorname{Ker} T(\rho)$ is in $\mathscr{M}\left(L_{1}\right)$ and, as was shown in the proof of Corollary (3.3), $I_{\rho} \cap \mathscr{Z}\left(L_{1}\right)=$ $I \cap \mathscr{Z}\left(L_{1}\right)$. Corollary (6.16) tells us that $I=I_{\rho}$. If $I_{\rho}=I_{\sigma}$ then $I_{\rho} \cap \mathscr{Z}\left(L_{1}\right)=I_{\sigma} \cap$ $\mathscr{Z}\left(L_{1}\right)$ and therefore $\left\{f \mid f \in \mathscr{Z}\left(L_{1}\right), f^{\wedge}(\rho)=0\right\}=\left\{f \mid f \in \mathscr{Z}\left(L_{1}\right), f^{\wedge}(\sigma)=0\right\}$. The fact that $\mathfrak{A}$ separates the points proves $\rho=\sigma$.

COROLlary (6.19). The above yields bijective maps, with a commutative diagram, between the following sets:

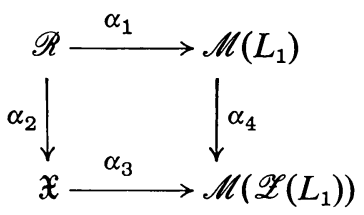

where $\alpha_{i}(i=1, \ldots, 4)$ are given by $\rho \rightarrow \operatorname{Ker} T(\rho), \rho \rightarrow \chi_{\rho} / d_{\rho}, \chi_{\rho} / d_{\rho} \rightarrow\left\{f \mid f^{\wedge}(\rho)=0\right\}$, $I \rightarrow I \cap \mathscr{Z}\left(L_{1}\right)$, respectively.

Proof. $\alpha_{1}, \alpha_{2}$, and $\alpha_{3}$ were shown to be bijective in Theorems (6.18), (4.1) of [10], and (6.4) respectively, while $\alpha_{4}$ was proven bijective in the course of the proof of Theorem (6.18), as was the commutativity of the diagram since this means that for $f \in \mathscr{Z}\left(L_{1}\right), f^{\wedge}(\rho)=0$ iff $T_{f}(\rho)=0$.

The next corollary seems to be the ring-theoretic analogue of Theorem (2.1) of [9].

Corollary (6.20). Each $I \in \mathscr{M}\left(L_{1}\right)$ is $\vee$-stable and has finite codimension.

Proof. By the above, $I=\operatorname{Ker} T(\rho)$, where $\rho \in \mathscr{R}$. Since $\rho$ is unitary, $T(\rho)$ is a *-algebra homomorphism and therefore $I$ is *-stable. Moreover, $d_{\rho}$ is finite and $T(\rho)\left(L_{1}\right)$ is the full matrix algebra, by the proof of Theorem (3.1). Thus we see that $\left[L_{1}: I\right]=d_{\rho}^{2}$.

This result implies that fin. rad. $\left(L_{1}\right)$ and st. rad. $\left(L_{1}\right)$ coincide in the case of $[Z]$-groups. Thus there is no distinction between Theorems (2.4) and (3.1) in that case. 


\section{REFERENCES}

1. J. Dixmier, Les $C^{*}$-algèbres et leurs représentations, Cahiers Scientifiques, fasc. 29, Gauthier-Villars, Paris, 1964. MR 30 \#1404.

2. N. Dunford and J. Schwartz, Linear operators. I: General theory, Pure and Appl. Math., vol. 7, Interscience, New York, 1958. MR 22 \#8302.

3. R. Godement, Analyse harmonique dans les groupes centraux. I: Fonctions centrales et caractères, C. R. Acad. Sci. Paris 225 (1947), 19-21. MR 9, 8.

4. - Analyse harmonique dans les groupes centraux. II: Formule d'inversion de Fourier, C. R. Acad. Sci. Paris 225 (1947), 221-223. MR 9, 134.

5. - L'analyse harmonique dans les groupes nonabéliens, Colloques Internationaux du C. N. R. S., no. 15, Paris, 1949. MR 11, 325.

6. - Mémoire sur la théorie des caractères dans les groupes localement compacts unimodulaires, J. Math. Pures Appl. (9) 30 (1951), 1-110. MR 13, 12.

7. - Les fonctions de type positif et la théorie des groupes, Trans. Amer. Math. Soc. 63 (1948), 1-84. MR 9, 327.

8. S. Grosser and M. Moskowitz, On central topological groups, Trans. Amer. Math. Soc. 127 (1967), 317-340. MR 35 \#292.

9. - Representation theory of central topological groups, Trans. Amer. Math. Soc. 129 (1967), 361-390. MR 37 \#5327.

10. —, Compactness conditions in topological groups. I, J. Reine Angew. Math. (to appear).

11. - Compactness conditions in topological groups. II, J. Reine Angew. Math. (to appear).

12. E. Hewitt, A survey of abstract harmonic analysis. Some aspects of analysis and probability, Surveys in Appl. Math., vol. 4, Wiley, New York and Chapman \& Hall, London, 1958, pp. 105-168. MR 21 \#2159.

13. E. Hewitt and K. A. Ross, Abstract harmonic analysis. I: Structure of topological groups. Integration theory, group representations, Die Grundlehren der math. Wissenschaften, Band 115, Academic Press, New York and Springer-Verlag, Berlin, 1963. MR 28 \#158.

14. I. Kaplansky, Primary ideals in group algebras, Proc. Nat. Acad. Sci. U.S.A. 35 (1949), 133-136. MR 10, 428.

15. H. Leptin and L. Robertson, [MAP]-groups are unimodular, Proc. Amer. Math. Soc. 19 (1968), 1079-1082.

16. L. Loomis, An introduction to abstract harmonic analysis, Van Nostrand, Princeton, N. J., 1953. MR 14, 883.

17. G. W. MACKEY, Functions on locally compact groups, Bull. Amer. Math. Soc. 56 (1960), 385-412. MR 12, 588.

18. - Infinite-dimensional group representations, Bull. Amer. Math. Soc. 69 (1963), 628-686. MR 27 \#3745.

19. - Induced representations of locally compact groups. I, Ann. of Math. (2) 55 (1952), 101-139. MR 13, 434.

20. Y. Misonou and M. Nakamura, Centering of an operator algebra, Tôhoku Math. J. (2) 3 (1951), 243-248. MR 14, 58.

21. M. A. Naĭmark, Normed rings, GITTL, Moscow, 1956; English transl., Noordhoff, Groningen, 1959. MR 19, 870; MR 22 \#1824.

22. I. E. Segal, The group algebra of a locally compact group, Trans. Amer. Math. Soc. 61 (1947), 69-105. MR 8, 438.

23. - An extension of Plancherel's formula to separable unimodular groups, Ann. of Math. (2) 52 (1950), 272-292. MR 12, 157. 
24. I. E. Segal, Algebraic integration theory, Bull. Amer. Math. Soc. 71 (1965), 419-489. MR 31 \#2642.

25. H. Sunouchi, An extension of the Plancherel formula to unimodular groups, Tôhoku Math. J. (2) 4 (1952), 216-230. MR 15, 399.

26. E. Thoma, Uber unitäre Darstellungen abzählbarer, diskreter Gruppen, Math. Ann. 153 (1964), 111-138. MR 28 \#3332.

27. —, Zur harmonischen Analyse klassenfiniter Gruppen, Invent. Math. 3 (1967), 20-42. MR 35 \#4343.

28. H. Yoshizawa, On some types of convergence of positive definite functions, Osaka Math. J. 1 (1949), 90-94. MR 11, 10.

UNIVERSITY OF MINNESOTA, MinNeAPOlis, Minnesota 55455

Columbia University, New York, New YoRK 10027 